\title{
A Unidirectional One-Dimensional Approach for Asphaltene Deposition in Large Length-to-Diameter Ratios Scenarios
}

Q. Guan ${ }^{1}$, J.C. Chai ${ }^{2}$, A. Goharzadeh ${ }^{1}$, F.M. Vargas ${ }^{3}$, S.L. Biswal ${ }^{3}$, W.G. Chapman ${ }^{3}$, M. Zhang ${ }^{4}$ and Y.F. Yap ${ }^{1, *}$

${ }^{1}$ Department of Mechanical Engineering, Khalifa University of Science and Technology, Sas Al Nakheel Campus, Abu Dhabi, UAE

${ }^{2}$ Department of Engineering and Technology, School of Computing and Engineering, University of Huddersfield, Huddersfield, UK

${ }^{3}$ Department of Chemical and Bio-molecular Engineering, Rice University, Houston, USA

${ }^{4}$ School of Energy and Power Engineering, Nanjing University of Science and Technology, Nanjing, P.R. China.

*Corresponding author:

Tel: +97126075175

Fax: +97126075200

Email address: yfatt@pi.ac.ae

Address: Department of Mechanical Engineering, Petroleum Institute, Khalifa University of Science and Technology, P.O. Box 2533, Abu Dhabi, UAE 


\section{A Unidirectional One-Dimensional One-Way Approach for Asphaltene Deposition in Large Length-to-Diameter Ratios Scenarios}

\section{ABSTRACT}

Asphaltene deposition in wellbores has been recognized as the cholesterol of petroleum for decades causing billions of dollars in losses to the oil and gas industry every year. This necessitates great efforts in precise and fast forecasting of the production problems induced by asphaltene deposition. From perspective of the large length-to-diameter ratios of wellbores and the unidirectional nature of the crude oil flow, this work presents a numerical procedure to predict the coupled velocity, pressure and concentration distribution in a transient one-dimensional one-way framework. This procedure is general-purpose for flow passages of large aspect ratios with the precipitation rate, aggregation rate and deposition rate embedded in known forms. In this numerical procedure, the governing equations are solved using the finite volume method on a regular mesh arrangement with fully implicit spatial and temporal schemes. For verification purpose, a few cases having exact solutions are studied. Then, application of the presented procedure to capillary asphaltene deposition is illustrated where good agreement is achieved between the simulation results and the experimental measurements. This case demonstrates that the proposed procedure can be used to investigate oilfield asphaltene problems and assist reservoir engineers in assessing the potential asphaltene deposition risk in wellbores.

21 Key Words: asphaltene deposition, large length-to-diameter ratios, one-dimensional one-way

\section{NONMENCLATURE}

$A_{c} \quad$ flow passage original cross-sectional area $\left(\mathrm{m}^{2}\right)$

$C_{\text {dis }} \quad$ concentration of the dissolved asphaltene $\left(\mathrm{kg} / \mathrm{m}^{3}\right)$

$C_{e q} \quad$ equilibrium asphaltene concentration $\left(\mathrm{kg} / \mathrm{m}^{3}\right)$

$C_{\text {pre }} \quad$ concentration of the precipitated asphaltene $\left(\mathrm{kg} / \mathrm{m}^{3}\right)$

$c_{p} \quad$ specific heat $(\mathrm{J} / \mathrm{kg} \cdot \mathrm{K})$

$D \quad$ flow passage diameter (m)

$f \quad$ underralaxation factor

$f_{D} \quad$ Darcy friction factor

$F_{w} \quad$ volumetric frictional force $\left(\mathrm{N} / \mathrm{m}^{3}\right)$

$k \quad$ rate coefficient (s-1)

$L \quad$ flow passage length (m)

M mass of the deposited asphaltene (kg)

$N \quad$ number of control volumes

$p \quad$ pressure $(\mathrm{Pa})$

$R \quad$ flow passage original radius (m)

$R_{\text {agg }} \quad$ aggregation rate of precipitated asphaltene $\left(\mathrm{kg} / \mathrm{m}^{3} \cdot \mathrm{s}\right)$

$R_{\text {dep }} \quad$ deposition rate of precipitated asphaltene $\left(\mathrm{kg} / \mathrm{m}^{3} \cdot \mathrm{s}\right)$

$R_{\text {pre }} \quad$ precipitation rate of dissolved asphaltene $\left(\mathrm{kg} / \mathrm{m}^{3} \cdot \mathrm{s}\right)$

Page 1 of $\mathbf{3 1}$ 


$\begin{array}{ll}r & \text { effective flow passage radius (m) } \\ S_{\phi} & \text { sources/sinks for the variable } \phi \\ S_{C} & \text { constant part of } S_{\phi} \\ S_{P} & \phi \text { dependent of } S_{\phi} \\ S_{T} & \text { energy sources/sinks } \\ S_{u} & \text { momentum sources/sinks } \\ S_{d i s} & \text { sources/sinks for } C_{\text {dis }} \\ S_{\text {pre }} & \text { sources/sinks for } C_{\text {pre }} \\ T & \text { temperature (K) } \\ t & \text { time (s) } \\ u & \text { velocity (m/s) } \\ x & \text { axial length (m) }\end{array}$

\section{Greek Symbols}

$\begin{array}{ll}\alpha & \text { crude oil volume fraction } \\ \delta & \text { deposit layer thickness }(\mathrm{m}) \\ \varepsilon & \text { surface roughness }(\mathrm{m}) \\ \mu & \text { crude oil dynamic viscosity }(\mathrm{Pa} \cdot \mathrm{s}) \\ \rho & \text { crude oil density }\left(\mathrm{kg} / \mathrm{m}^{3}\right) \\ \rho_{\text {dep }} & \text { asphaltene deposit density }\left(\mathrm{kg} / \mathrm{m}^{3}\right) \\ \widetilde{\rho} & \text { "density" of the dependent variable } \\ \tau & \text { local shear stress }\left(\mathrm{N} / \mathrm{m}^{2}\right) \\ \phi & \text { generic dependent variable }\end{array}$

\section{Subscripts}

$\begin{array}{ll}\text { agg } & \text { asphaltene aggregation } \\ \text { asph } & \text { asphaltene component } \\ \text { dep } & \text { asphaltene deposition } \\ \text { dis } & \text { asphaltene re-dissolution } \\ \text { i } & \text { downstream interface } \\ \text { i-1 } & \text { upstream interface } \\ \text { in } & \text { inlet } \\ P & \text { current position } \\ \text { pre } & \text { asphaltene precipitation } \\ U & \text { upstream position }\end{array}$

\section{Superscripts}

$\begin{array}{ll}n & \text { current time step } \\ n-1 & \text { previous time step } \\ 0 & \text { previous time step } \\ * & \text { most current value }\end{array}$




\section{INTRODUCTION}

Asphaltene depositions can occur during the oil recovery process in the near wellbore regions and in the wellbores, during transportation in the pipelines, and during processing in the separators (Akbarzadeh et al., 2007; Hoepfner et al., 2013; Mansoori, 2010). Crude oil's pressure, temperature and composition change while it travels from the reservoir to the earth surface. Due to these changes, the dissolved asphaltene can precipitate and deposit on the wall of the wellbore. The deposits lead to reduction in flow areas resulting in the decrease in production. In severe cases, the deposits block the wellbore completely and thus cause the complete loss of production. The well is then shut down to facilitate asphaltene removal; usually accomplished using various chemicals. The associated preventive and remedial measures incur tremendous financial losses (Creek, 2005).

11 Due to the depth of oil reservoirs, the wellbore's length-to-diameter $(L / D)$ ratio is extremely large. 12 A typical reservoir depth of $4.5 \mathrm{~km}$ and a wellbore diameter of $10 \mathrm{~cm}$ results in an $L / D$ ratio of 45,000. As a result, full Computational Fluid Dynamics (CFD) solution is impractical even for single-phase flows. There is a need for a simplified asphaltene deposition, aggregation, precipitation and transport procedure. This procedure must be capable of modelling asphaltene precipitation, aggregation, deposition and the complex interactions between these when oil is transported through the wellbore.

Chapman and co-workers (Kurup et al., 2011; Kurup et al., 2012; Wang et al., 2004) presented a one-dimensional approach to model asphaltene deposition in large $L / D$ ratio wellbores. In their approach, the crude oil velocities are specified through the well-known fully-developed flows' expressions. The transient concentration of the dissolved asphaltene is then solved over the wellbore until the specified time has been reached. The precipitation of asphaltene, aggregation of asphaltene and subsequent deposition of asphaltene change the concentration of the dissolved asphaltene. The Perturbed Chain-Statistical Association Fluid Theory (PC-SAFT) equation of state (EOS) was used to predict the thermodynamic properties of crude oil. Once the concentration of the precipitated asphaltene is predicted, the asphaltene fluxes normal to and near the wellbore's wall are obtained. Asphaltene depositions on the wall are assumed to be $100 \%$, i.e. once a precipitated asphaltene particle is in contact with the wall, it is deposited. From these deposition fluxes, the evolution of asphaltene deposited on the wall is obtained.

Ramirez-Jaramillo et al. (2006) developed a multiphase multicomponent hydrodynamic model to model asphaltene deposition in production pipelines. In their model, a thermodynamic sub-module is designed to predict the condition and the amount of precipitated asphaltene particle using the SAFT-VR (Statistical Associating Fluid Theory for Potentials of Variable Range) EOS. Besides, a series of semi-empirical correlations for multiphase flow are utilized to model particle transport in different flow regimes and further to predict the mass of asphaltene deposit as a function of time and axial position.

Using experimental data, Soulgani et al. (2009) fitted a correlation for asphaltene deposition rate as a function of oil velocity, surface temperature and precipitated asphaltene particle concentration. The obtained asphaltene deposition rate correlation was coupled with a thermodynamic asphaltene precipitation model to predict precipitated asphaltene particle concentration and a hydrodynamic well model based on the correlation of Beggs and Brill (1973) to determine pressure, temperature, velocity and density. 
Following a similar modeling idea, Kor and Kharrat (2016) adopted the correlation of Mukherjee and Brill (1985) to hydrodynamically model the multiphase flows, the correlations of Ramey $\mathrm{Jr}$ (1962) and Hasan and Kabir (1991) to perform radial heat transfer calculations, the characterization method of Nghiem et al. (1993) to determine asphaltene precipitated particle concentration, and the model of Jamialahmadi et al. (2009) to calculate asphaltene deposition rate.

Based on the material balance of asphaltene species, Vargas et al. (2010) developed a numerical model to simultaneously consider asphaltene precipitation, aggregation, particle transport and deposition. In this model, a thermodynamic module was employed to determine asphaltene precipitation rate using PC-SAFT EOS. Particle transport is assumed driven by combined advection-diffusion. Asphaltene aggregation and deposition are treated as pseudo-first order reactions. By solving this only species conservation equation, a dimensionless deposition flux can be obtained to quantify the extent of asphaltene deposition problem.

In these above studies, either fully-developed flows' expressions are used to represent the velocities in wellbores or hydrodynamic models with empirical correlations are used to model the oil flow. Coupling the velocity and pressure fields to the growing deposit layer is however not considered. This is mainly due to the modeling complication of a moving boundary. In fact, with the evolution of the deposit layer, the effective flow area is gradually reduced which alters the flow fields in wellbores. Once the temperature and pressure condition is changed, the thermodynamic state of asphaltene in oil changes accordingly. Hence, asphaltene precipitation and deposition are eventually affected, which in turn determines the deposit thickness. In short, all the phenomena involved are tightly coupled. To the authors' best knowledge, there is no existing work available in the open literature accounting for this coupling effect. The present article aims to present an approach to predict the coupled velocity, pressure and concentration distributions in a transient one-dimensional framework for large $L / D$ ratio scenarios. The energy equation and other scalar equations may also affect the deposition process. Similar to the concentration equations, the solutions of these scalar equation(s) can be incorporated without new concepts and is not detailed in this article.

\section{Ingredients of an Asphaltene Deposition Solution Method}

Figure 1 shows the ingredients of an asphaltene deposition model. There are two parts in this model, namely, the thermodynamic model to characterize the crude oil and the CFD part, which besides predicting the fluid flow also makes use of the results of the thermodynamic model to predict asphaltene deposition. Chapman and co-workers (Kurup et al., 2011; Kurup et al., 2012; Wang et al., 2004) employed the PC-SAFT EOS to characterize the crude oil. Although thermodynamic characterizations are critical to an asphaltene deposition model, it is not the focus of this article. In this article, we focus our attention on specific portions of the CFD part of an asphaltene deposition model. As such, it is assumed that the precipitation rate, aggregation rate and deposition rate are known, given or modelled using simple relations. Our proposed procedure makes use of these rates to predict the asphaltene deposition front for demonstration purposes. The procedure is general in that it can be used with other forms of rate expressions without reformulation.

\section{Simplifications for Large Length-to-Diameter Ratios}

40 To facilitate the large $L / D$ ratios encountered in wellbores, we reformulate our governing 41 differential equations to situations where (i) there exists a predominant flow direction (i.e. the flow is unidirectional and there is no reverse flow in the wellbore), (ii) the flow Peclet number Pe is large so that downstream conditions are affected by upstream conditions and happenings at a downstream 
point do not affect upstream locations, and (iii) the upstream pressure field is not affected by the

2 downstream pressure field. When the above conditions are met, the main flow direction becomes a "one-way" coordinate (Patankar, 1980). We can then employ a marching procedure starting from the inlet as upstream conditions to determine downstream conditions.

\section{Outline of the Present Article}

The remainder of this article is divided into five sections. Section 2 introduces the main features of the governing equations. Section 3 details the discretization and solution procedures. In Section 4, the auxiliary variables used in this article are discussed. In Section 5, the capability of the proposed numerical procedure is demonstrated through investigating several problems with either exact solutions or experimental measurements. Finally, a few concluding remarks are also given.

\section{MAIN FEATURES OF THE GOVERNING EQUATIONS}

\section{Background}

In addition to the simplifications for large $L / D$ ratios mentioned above, we further simplify our formulation to one-dimensional flow as shown in Figure 2. The procedure presented in this article will work for all the situations depicted in Figure 2. Figure 2a shows a one-dimensional situation with constant cross-sectional area. When the fluid density is constant, the axial flow velocity remains constant throughout the flow passage. Figure $2 \mathrm{~b}$ shows a one-dimensional flow through a curved duct. Again, the axial flow velocity remains constant if both the cross-sectional area and the fluid density are unchanged. Flow reversal can occur when fluid tries to flow through a curved duct. The procedure proposed in this article will work if the mean velocity at every cross-section is unidirectional flowing from the inlet towards the outlet. Figure 2c shows flow through a contracting channel. In this situation, the mean velocity increases if the fluid density is constant. This ability to model flow in channel with changing cross-sectional area paths the way to model flow with asphaltene deposition where the flow area reduces as deposit forms (Figure 2d).

\section{Governing Equations}

The unidirectional one-dimensional one-way continuity, momentum and concentration equations are summarized here. Unidirectional implies that throughout the whole flow passage, flow is in the same direction. One-dimensional indicates quantities vary in the axial direction only. One-way assumes axial diffusion is negligible. The derivation of the continuity equation is given in Appendix A for completeness.

Continuity:

$$
\frac{\partial}{\partial t}(\alpha \rho)+\frac{\partial}{\partial x}(\alpha \rho u)=-\alpha R_{d e p}
$$

Momentum:

$$
\frac{\partial}{\partial t}(\alpha \rho u)+\frac{\partial}{\partial x}(\alpha \rho u u)=-\alpha \frac{d p}{d x}-\alpha F_{w}+\alpha S_{u}
$$

Concentration of the dissolved asphaltene $C_{\text {dis: }}$ : 


$$
\frac{\partial}{\partial t}\left(\alpha C_{d i s}\right)+\frac{\partial}{\partial x}\left(\alpha u C_{d i s}\right)=-\alpha R_{p r e}+\alpha S_{d i s}
$$

Concentration of the precipitated asphaltene $C_{\text {pre: }}$ :

$$
\frac{\partial}{\partial t}\left(\alpha C_{p r e}\right)+\frac{\partial}{\partial x}\left(\alpha u C_{p r e}\right)=\alpha R_{p r e}-\alpha R_{\text {agg }}-\alpha R_{\text {dep }}+\alpha S_{\text {pre }}
$$

2 Energy:

$$
\frac{\partial}{\partial t}\left(\alpha \rho c_{p} T\right)+\frac{\partial}{\partial x}\left(\alpha \rho c_{p} u T\right)=\alpha S_{T}
$$

where $t$ is the time, $\alpha$ is the crude oil volume fraction, $x$ is the axial coordinate, $\rho$ is the density of crude oil, $u$ is the average axial velocity, $R_{d e p}$ is the deposition rate of the precipitated asphaltene, $p$ is the average pressure at a cross-section, $F_{w}$ is the volumetric frictional force, $S_{u}$ is additional momentum sources/sinks, $C_{\text {dis }}$ is the concentration of the dissolved asphaltene, $R_{\text {pre }}$ is the precipitation rate of the dissolved asphaltene, $S_{\text {dis }}$ is additional sources/sinks for $C_{d i s}, C_{p r e}$ is the concentration of the precipitated asphaltene, $R_{a g g}$ is the aggregation rate of the precipitated asphaltene, $S_{\text {pre }}$ is additional sources/sinks for $C_{p r e}, C_{p}$ is the specific heat, $T$ is the temperature, and $S_{T}$ is the volumetric energy sources/sinks. The total sources/sinks (i.e. $\alpha R_{\text {pre }}$ ) are per unit total volume. The volumetric sources/sinks (i.e. $R_{\text {pre }}$ ) are per unit fluid volume.

A general transport equation for a generic dependent variable $\phi$ can be written as

$$
\frac{\partial}{\partial t}(\alpha \tilde{\rho} \phi)+\frac{\partial}{\partial x}(\alpha \tilde{\rho} u \phi)=\alpha S_{\phi}
$$

where $\tilde{\rho}$ is the "density" and $S_{\phi}$ is the sources/sinks for the dependent variable per unit total (oil + deposit) volume. The meanings of $\tilde{\rho}$ and $S_{\phi}$ for various dependent variables are shown in Table 1.

\section{Remarks on the Governing Equations}

The governing equations (eqs $1-5$ ) are written without reference to the cross-sectional shape of the flow passage. As such, these equations and the numerical method presented in this article can be used for flow passage of different shapes. The exact expressions to calculate certain quantities may require knowledge of the cross-sectional shape, but do not affect the applicability of the current solution method.

Due to the one-dimensional nature of the procedure, two observations can be noticed. Firstly, there is only one velocity, which is the axial velocity, at every cross-section of the flow passage. As a result, the transverse velocity is zero and does not appear in the governing equations (eqs $1-5$ ). Secondly, the diffusion in the transverse direction, which depends on the velocity gradient of the axial velocity in the transverse direction, is also zero and does not appear in the momentum (eq 2) and other transport equations (eqs $3-5$ ).

As a result of the one-way nature of the flow, the axial diffusion is also negligible and does not appear in the transport equations (eqs $2-5$ ). The effects of the shear stress on the flow are accounted through the volumetric frictional force $F_{w}$ in the momentum equation (eq 2). Similar 
effects of the axial gradient on the transport of other dependent variable can also be incorporated in the same manner.

The exact expressions of the various sources/sinks, namely, $R_{\text {dep }}, R_{\text {pre }}$ and $R_{\text {agg }}$ affect the amount of, the rate of and the shape of the deposition layer amongst others. The solution procedure is designed to work with various forms of these sources/sinks. In this article, a general-purpose solution method for the governing equations (eqs $1-5$ ) is presented without reference to the exact forms of these sources/sinks. The exact forms of these sources/sinks will be shown when the general-purpose solution method is applied to different problems.

As the purpose of this article is to present a unidirectional, one-dimensional, one-way method to model deposition, the temperature changes which affect the rate of precipitation, the rate of deposition, the density, the viscosity and etc. are not considered. The flow is assumed to be isothermal and thus the energy equation is not solved. The temperature field can be readily obtained by solving the energy equation (eq 5). As there are no new numerically related concepts in the solution of the energy equation, it can be added as an additional scalar equation and solved as part of a larger set of governing equations iteratively using the general-purpose solution method presented in the article. For completeness, the energy equation is shown in eq 5.

\section{Crude Oil Volume Fraction}

The crude oil volume fraction $\alpha$ appearing in eqs $1-5$ deserves some attentions. As deposit forms, the flow area reduces. The open flow area available for the crude oil is shown in Figure 3. The ratio of this open flow area to the original cross-sectional area of the pipe is defined as the crude oil volume fraction $\alpha$. An approach to calculate $\alpha$ will be discussed in the next section. As mentioned above, the proposed method is applicable to flow passages of different shapes, a pipe (circular cross-section) is used in Figure 3 for illustration purposes only.

\section{DISCRETIZATION EQUATIONS AND SOLUTION PROCEDURE}

\section{Control Volumes, Grid Points and Storage Locations}

For discretization purposes, the flow passage is divided into $N$ non-overlapping control volumes (CVs) as shown in Figure 4. All variables are stored at the CV boundaries; labelled with either filled or open circles. Internal nodes are depicted using filled circles, while boundary grid points are identified using open circles. As a result, there is a total of $N+1$ sequentially numbered nodes, i.e. two boundary nodes and $N-1$ internal nodes. Note that the sizes of the CVs need not be uniform.

\section{Discretization Equation for the General Transport Equation}

A discretization equation for the general transport equation (eq 6) can be obtained by integrating

where $A_{c}$ is the original cross-sectional area of the flow passage. The fully-implicit scheme where the value of $\phi$ at time $t+\Delta t$ prevails over the time step $\Delta t$ is used to discretize the time variation. 
1 implicit" spatial differencing is also used to discretize the spatial variation between $i-1$ and $i$.

2 Using these two differencing practices, eq 7 becomes

$$
\frac{\left(\alpha \tilde{\rho} \phi A_{c} \Delta x\right)_{i}^{n}-\left(\alpha \tilde{\rho} \phi A_{c} \Delta x\right)_{i}^{n-1}}{\Delta t}+\left[\left(\alpha \tilde{\rho} u \phi A_{c}\right)_{i}^{n}-\left(\alpha \tilde{\rho} u \phi A_{c}\right)_{i-1}^{n}\right]=\left(\alpha S_{\phi}\right)_{i}^{n} A_{c} \Delta x
$$

3 To further simplify future writing, we shall drop the superscript $n$ from current time step values and

4 replace $n-1$ with 0 for the values from the previous time step. We shall refer to the current position 5 of interest $i$ as $P$ and replace the subscript for the upstream location $i-1$ with $U$ to denote an 6 upstream location as shown in Figure 4. Using these practices, eq 8 simplifies to

$$
\frac{(\alpha \tilde{\rho} \phi \Delta x)_{P}-(\alpha \tilde{\rho} \phi \Delta x)_{P}^{0}}{\Delta t}+(\alpha \tilde{\rho} u \phi)_{P}-(\alpha \tilde{\rho} u \phi)_{U}=\alpha\left[\left(S_{C}+S_{P} \phi_{P}\right)\right] \Delta x
$$

$7 \quad$ Note that in eq 9, the source term was further written as (Patankar, 1980)

$$
S_{\phi}=S_{C}+S_{P} \phi_{P}
$$

8 A discretization equation for $\phi$ can then be written as

$$
a_{P} \phi_{P}=a_{U} \phi_{U}+a_{P}^{0} \phi_{P}^{0}+b_{\phi}
$$

9 where

$$
\begin{gathered}
a_{U}=(\alpha \tilde{\rho} u)_{U} \\
a_{P}^{0}=\frac{(\alpha \tilde{\rho} \Delta x)_{P}^{0}}{\Delta t} \\
b_{\phi}=\alpha S_{C} \Delta x \\
a_{P}=(\alpha \tilde{\rho} u)_{P}+\frac{(\alpha \tilde{\rho} \Delta x)_{P}}{\Delta t}-\alpha S_{P} \Delta x
\end{gathered}
$$

10 In eqs 11 and 12, $a_{U}$ is the neighbour coefficient in space, $a_{P}^{0}$ is the "neighbour" coefficient in time,

$11 b_{\phi}$ is the sources/sinks, $S_{C}$ is the constant part of the sources/sinks and $S_{P}$ is the $\phi$ dependent part 12 of the sources/sinks.

13 Discretization Equations for Continuity, Momentum and Concentration Equations

14 Using eq 9 and Table 1, the continuity equation can be written as

$$
\frac{(\alpha \rho \Delta x)_{P}-(\alpha \rho \Delta x)_{P}^{0}}{\Delta t}+(\alpha \rho u)_{P}-(\alpha \rho u)_{U}=-\alpha R_{d e p, P} \Delta x
$$

15 The discretized form of eq 13 can be written as

Page 8 of $\mathbf{3 1}$ 


$$
a_{P} u_{P}=a_{U} u_{U}+b_{1}
$$

1 where

$$
\begin{gathered}
a_{U}=(\alpha \rho)_{U} \\
b_{1}=\frac{(\alpha \rho \Delta x)_{P}^{0}-(\alpha \rho \Delta x)_{P}}{\Delta t}-\alpha R_{d e p, P} \Delta x \\
a_{P}=(\alpha \rho)_{P}
\end{gathered}
$$

2 Note that the subscript "1" is used in eq 14 as $\phi=1$ for the continuity equation. Due to the one3 dimensional nature of the formulation, a continuity satisfying velocity is obtained from eqs 14 and 4 15. The downstream pressure is then calculated using the momentum equation and the continuity satisfying velocity to obtain a pressure field that ensures continuity and momentum satisfying velocity and pressure fields. Using eqs 2 and 9, the downstream pressure $p_{P}$ can be obtained from the momentum equation as

$$
p_{P}=p_{U}+a_{U} u_{U}-a_{P} u_{P}+a_{P}^{0} u_{P}^{0}+b_{u}
$$

8 where

$$
\begin{gathered}
a_{U}=\frac{(\alpha \rho u)_{U}}{\alpha_{P}} \\
a_{P}=\frac{(\alpha \rho u)_{P}}{\alpha_{P}}+\frac{(\alpha \rho \Delta x)_{P}}{\alpha_{P} \Delta t} \\
a_{P}^{0}=\frac{(\alpha \rho \Delta x)_{P}^{0}}{\alpha_{P} \Delta t} \\
b_{u}=\left(S_{u}-F_{w}\right)_{P} \Delta x
\end{gathered}
$$

9 Using eqs 9, 11 and Table 1, the discretized forms of the concentration of the dissolved asphaltene, 10 concentration of the precipitated asphaltene, energy and other scalar variables can also be written.

11 A solution algorithm must be setup to solve the set of discretization equations which include the 12 continuity (eq 14), momentum (eq 16), concentration of the dissolved asphaltene (eq 11), 13 concentration of the precipitated asphaltene (eq 11), energy (eq 11), and other scalar variables (eq 14 11). We have not discussed the forms and/or calculation procedures for some "auxiliary" variables 15 appearing in eqs $14-17$. This is by choice as we do not want to clutter the discussions of the general-purpose solution method with detailed on the evaluations of problem-specific "auxiliary" variables. However, we shall discuss these at an appropriate juncture.

\section{Crude Oil Volume Fraction}


1 In the solution process, it is important to be able to calculate the crude oil volume fraction $\alpha$ from

2 the latest value of the deposition rate of the precipitated asphaltene $R_{d e p}$. The change in the mass of

3 the deposited asphaltene in a control volume over the a time step from $t$ to $t+\Delta t$ is

$$
M_{d e p, P}-M_{d e p, P}^{0}=\int_{t}^{t+\Delta t}\left(\int_{x_{i-1}}^{x_{i}} \alpha_{P} R_{d e p, P} A_{c} d x\right) d t
$$

4 where $M_{d e p, P}$ is the mass of the deposited asphaltene of the current time step, $M_{d e p, P}^{0}$ is the mass of the deposited asphaltene of the previous time step. This change in mass can be expanded as

$$
\rho_{\text {dep }, P}\left(1-\alpha_{P}\right) A_{c} \Delta x-\rho_{d e p, P}^{0}\left(1-\alpha_{P}^{0}\right) A_{c} \Delta x=\alpha_{P} R_{d e p, P} A_{c} \Delta x \Delta t
$$

$6 \quad$ The above equation can be simplified to

$$
\alpha_{P}=\frac{\rho_{d e p, P}-\rho_{d e p, P}^{0}}{\rho_{d e p, P}+R_{d e p, P} \Delta t}+\frac{\rho_{d e p, P}^{0}}{\rho_{d e p, P}+R_{d e p, P} \Delta t} \alpha_{P}^{0}
$$

Some comments on eq 20 is in order. If the deposition rate $R_{d e p, P}$ and the density of the deposit $\rho_{\text {dep, } P \text {, }}$ both of the current time step, are known, the crude oil fraction at $t+\Delta t$ can be determined from eq 20 without further iterations. The total change in the crude oil fraction over the time step is captured in eq 20. During the initial stages of solution and when other variables are guessed values, it may be beneficial to slow down the change in the volume fraction update. Underrelaxation is introduced to stabilize the iteration process. Using eq 20, the maximum possible change in the crude oil fraction is

$$
\Delta \alpha_{\max , P}=\alpha_{P}-\alpha_{P}^{*}=\frac{\rho_{d e p, P}-\rho_{d e p, P}^{0}}{\rho_{d e p, P}+R_{d e p, P} \Delta t}+\frac{\rho_{d e p, P}^{0}}{\rho_{d e p, P}+R_{d e p, P} \Delta t} \alpha_{P}^{0}-\alpha_{P}^{*}
$$

where $\alpha_{P}^{*}$ is the most current value of $\alpha_{P}$. These changes are "slowed" down by introducing an underrelaxation factor. The final expression to update the crude oil fraction can then be written as

$$
\alpha_{P}=\alpha_{P}^{*}+f_{\alpha} \Delta \alpha_{\max , P}=f_{\alpha}\left(\frac{\rho_{\text {dep }, P}-\rho_{d e p, P}^{0}}{\rho_{\text {dep }, P}+R_{d e p, P} \Delta t}+\frac{\rho_{d e p, P}^{0}}{\rho_{\text {dep }, P}+R_{d e p, P} \Delta t} \alpha_{P}^{0}\right)+\left(1-f_{\alpha}\right) \alpha_{P}^{*}
$$

where $f_{\alpha}$ is the underrelaxation factor.

\section{Underrelaxation of the Dependent Variables}

Iterations are employed to resolve the nonlinearities in the governing equations and also couplings between dependent variables. In many situations, underrelaxation is needed for the solution to converge. The maximum possible change in the general variable $\phi$ can then be written as

$$
\Delta \phi_{\max , P}=\phi_{P}-\phi_{P}^{*}
$$

where $\phi_{P}^{*}$ is the most current value of $\phi_{P}$. Substituting eq 11 into eq 23, the maximum changes can be written as 


$$
\Delta \phi_{\max , P}=\frac{a_{U} \phi_{U}+a_{P}^{0} \phi_{P}^{0}+b_{\phi}}{a_{P}}-\phi_{P}^{*}
$$

1 These changes are slowed down by introducing an underralaxation factor $f_{\phi}$ and can be written as

$$
\phi_{P}=\phi_{P}^{*}+f_{\phi} \Delta \phi_{\text {max }, P}=f_{\phi}\left(\frac{a_{U} \phi_{U}+a_{P}^{0} \phi_{P}^{0}+b_{\phi}}{a_{P}}\right)+\left(1-f_{\phi}\right) \phi_{P}^{*}
$$

2 The final discretization equation for $\phi$ with underrelaxation can then be written as

$$
\frac{a_{P}}{f_{\phi}} \phi_{P}=a_{U} \phi_{U}+a_{P}^{0} \phi_{P}^{0}+b_{\phi}+\left(1-f_{\phi}\right) \frac{a_{P}}{f_{\phi}} \phi_{P}^{*}
$$

3 where

$$
\begin{gathered}
a_{U}=(\alpha \tilde{\rho} u)_{U} \\
a_{P}^{0}=\frac{(\alpha \tilde{\rho} \Delta x)_{P}^{0}}{\Delta t} \\
b_{\phi}=\alpha S_{C} \Delta x \\
a_{P}=(\alpha \tilde{\rho} u)_{P}+\frac{(\alpha \tilde{\rho} \Delta x)_{P}}{\Delta t}-\alpha S_{P} \Delta x
\end{gathered}
$$

\section{Solution Algorithm}

The solution algorithm depends on what are known at the inlet. In this article, the inlet mass flow rate, inlet concentrations and properties at the inlet plane are known. The overall solution procedure for the proposed method can be summarized as follows:

1. Specify the inlet values at $i=1$ and the initial conditions at the initial time.

2. Advance the time step to $t+\Delta t$.

a. Advance the spatial location to $i+1$ and begin an inner iteration loop.

i. Solve for the velocity $u$ using the continuity equation (eq 14 ) with the latest $R_{d e p, P}$ and $\alpha_{P}$.

ii. Solve for the pressure $p$ using the momentum equation (eq 16).

iii. Solve for concentrations $C_{d i s}$ and $C_{\text {pre }}$ using eqs 11 and 12.

iv. Calculate $R_{d e p, P}, R_{p r e, P}, R_{a g g, P}$ and $F_{w, P}$.

v. $\quad$ Solve for the crude oil volume fraction $\alpha_{P}$ using eq 22 .

vi. Repeat i. to v. until the solutions converge, then exit the inner iteration loop.

b. Update the previous time step values with the current time step solutions

c. Repeat a. and b. until $i=N+1$, then exit the spatial sweep.

3. Set $i=1$ and repeat 2 . until the desired time is attained. 
1 We presented a discretization approach and a solution algorithm. Both the discretization approach 2 and the solution algorithm are generic and can be applied to problems that can be described using 3 the unidirectional, one-dimensional, one-way formulation presented in this article. The expressions

4 specific to different situations will be discussed next.

\section{AUXILLARY VARIABLES}

6 Various auxiliary variables used in this article are discussed in this section and they are used to 7 demonstrate the solution procedure. Their exact forms can be changed according to the actual

8 situations without reformulation of the general-purpose solution procedure.

\section{$9 \quad$ Frictional Force}

10 The frictional force $F_{w}$ for a control volume in eq 2 is evaluated using the friction factor through

$$
\int_{x_{i-1}}^{x_{i}} \alpha F_{w} A_{c} d x=\int_{x_{i-1}}^{x_{i}} \tau P_{i} d x
$$

11 where $\tau$ is the shear stress on the deposition surface and $P_{i}$ is the perimeter of the deposition surface.

12 For pipe flow with circumferentially uniform deposit, the perimeter is

$$
P_{i}=2 \pi r_{i}
$$

where $A_{i}$ is the available flow area. The Reynolds number is defined as

$$
\operatorname{Re} \equiv \frac{\rho u D_{h}}{\mu}
$$

where $\mu$ is the crude oil viscosity. 
Auxiliary variables such as $R_{d e p}, R_{p r e}, R_{a g g}$ and others are crucial to accurate predictions. The procedure presented in this article is general and the readers can adopt it with any form of auxiliary variables of his/her choice. The use of these auxiliary variables is demonstrated next.

\section{RESULTS AND DISCUSSIONS}

\section{Background}

In asphaltene deposition modelling, the deposition rate determines the deposition thickness which in turn changes the velocity and pressure. The above is of course coupled with the aggregation rate, precipitation rate, concentration of the dissolved asphaltene, concentration of the precipitated asphaltene, and energy. The numerical procedure presented in this work has been implemented into free-format Fortran (Fortran 90 ) codes with the latest Intel ${ }^{\circledR}$ Fortran Compiler. In this section, four problems are investigated based on the newly developed codes to verify, validate and demonstrate the proposed numerical procedure.

\section{Problem 1}

Problem Description: For flow field calculation, first we consider the flow in a long circular pipe of constant cross section. This case aims at testing the ability of the proposed numerical procedure to predict the correct pressure drop along this pipe.

Properties and Sources/Sinks: For demonstration purpose, the length $L$ and diameter $D$ of the pipe are set to $10 \mathrm{~m}$ and $0.02 \mathrm{~m}$ respectively. The fluid having a density $\rho$ of $820 \mathrm{~kg} / \mathrm{m}^{3}$ and viscosity $\mu$ of $3.95 \times 10^{-3} \mathrm{~kg} / \mathrm{m} \cdot \mathrm{s}$ flows into the pipe with an inlet velocity $u_{\text {in }}$ of $0.2 \mathrm{~m} / \mathrm{s}$.

Exact Solutions: The theoretical pressure drop across this pipe can be calculated from (Brown, 2000)

$$
\Delta p=f_{D} \frac{L}{D} \frac{\rho u^{2}}{2}
$$

Since $\operatorname{Re}=\rho u D / \mu \approx 830.3797<2300$, the flow is a laminar flow. Thus, the Darcy friction factor $f_{D}$ is evaluated as $f_{D}=64 / \operatorname{Re} \approx 0.07707$. Finally, $(\Delta p)_{\text {theoretical }}=632.00 \mathrm{~Pa}$.

Discussions of Results: As shown in Figure 5, a pressure drop of $(\Delta p)_{\text {predicted }}=632.00 \mathrm{~Pa}$ is predicted by the proposed numerical procedure using both 20 and $100 \mathrm{CVs}$. This prediction is exactly identical to the theoretical one. Besides, a constant velocity of $u=0.2 \mathrm{~m} / \mathrm{s}$ is also predicted along the entire pipe, consistent with the theoretical understanding. Then, the Reynolds number is increased to $\mathrm{Re}=8303.7975$ so that the oil flow is in a turbulent regime. Instead, the Darcy friction factor $f_{D}$ is calculated from the Colebrook relation (Colebrook et al., 1939). Again, the theoretical pressure drop $(\Delta p)_{\text {theoretical }}=26621.81 \mathrm{~Pa}$ is successfully predicted.

Remarks: This problem shows the capability of the proposed procedure in predicting pressure drop for both laminar and turbulent flows in a circular pipe of constant flow area and with a length-todiameter ratio of 500 .

\section{Problem 2}

Problem Description: In general, the cross section of a pipe is not always constant, for example, in the presence of deposits. The procedure presented in this article should also be able to predict the flow fields (both velocity and pressure) in such a pipe. The schematic of a pipe with deposits is Page 13 of 31 
shown in Figure 6 . The pipe is considered to have a cross section of diameter $D_{1}$, while deposits are formed in the second and third pipe sections, reducing the diameter to respectively $D_{2}$ and $D_{3}$.

Properties and Sources/Sinks: For demonstration purpose, the diameters of these three pipe sections are taken as $0.02 \mathrm{~m}, 0.01 \mathrm{~m}$ and $0.007 \mathrm{~m}$ respectively. The corresponding pipe lengths are $10 \mathrm{~m}, 5 \mathrm{~m}$ and $1 \mathrm{~m}$ respectively. The fluid having a density $\rho$ of $820 \mathrm{~kg} / \mathrm{m}^{3}$ and viscosity $\mu$ of $3.95 \times 10^{-3} \mathrm{~kg} / \mathrm{m} \cdot \mathrm{s}$ enters the pipe with a velocity of $u_{i n}=0.2 \mathrm{~m} / \mathrm{s}$. Note that in this case the deposit layer thickness is fixed, and no subsequent deposition takes place.

Exact Solutions: The major pressure drop caused by friction can be determined theoretically section by section using eq 34, as tabulated in Table 3. Summing up the major pressure drops in these three sections gives $(\Delta p)_{\text {major,theoretical }}=12999.67 \mathrm{~Pa}$. Besides, using the empirical correlations eq 35 given by King et al. (1984), the theoretical minor pressure drop due to sudden contraction is calculated as $(\Delta p)_{\text {minor,theoretical }} \approx 61.99+119.39 \mathrm{~Pa}=181.38 \mathrm{~Pa}$. Finally, the total theoretical pressure drop is $(\Delta p)_{\text {theoretical }}=13181.05 \mathrm{~Pa}$.

$$
\begin{gathered}
(\Delta p)_{\text {minor }, S C}=K_{S C} \frac{\rho u_{s}^{2}}{2} \\
K_{S C}=\left\{\begin{array}{l}
0.42\left(1-\frac{d_{s}^{2}}{d_{l}^{2}}\right)^{2}, \text { for } \frac{d_{s}}{d_{l}} \leq 0.76 \\
\left(1-\frac{d_{s}^{2}}{d_{l}^{2}}\right)^{2}, \text { for } 0.76<\frac{d_{s}}{d_{l}} \leq 1
\end{array}\right.
\end{gathered}
$$

where $u_{s}$ is the velocity in the smaller pipe section, $d_{s}$ and $d_{l}$ are the diameters of the smaller and larger pipe sections respectively.

Discussions of Results: Based on the Re calculated (Table 3), the flow in the first and second sections is laminar, whereas it is turbulent in the third section. The total pressure drop across the whole pipe predicted by the proposed numerical procedure is slightly larger $(\Delta p)_{\text {predicted }}=13669.68$. Pa, i.e. about $3.72 \%$ of the theoretical pressure drop. Considering the use of empirical correlations in the calculation of theoretical minor pressure drop, this discrepancy is reasonable and acceptable. By adopting 100, 50 and $10 \mathrm{CVs}$ respectively in these three pipe sections, the mesh-independent fluid volume fraction, velocity and pressure are shown in Figure 7. It is clearly seen that the change in the cross-sectional area significantly affects the velocity and pressure fields along the pipe.

Remarks: This problem shows that the numerical procedure proposed in the article is able to predict the effect of the presence of a fixed deposit layer on the flow fields in a pipe with an $L / D$ ratio of 800 .

\section{Problem 3}

Problem Description: The objectives of this problem is to demonstrate the ability of the procedure to capture the oil volume fraction $\alpha$ (or the deposition thickness $\delta$ ) and the fluid velocity when the deposition rate is known and specified. For demonstration purpose, we consider a situation where the oil flows at a constant volumetric flowrate of $11.7 \mathrm{ml} / \mathrm{hr}$ (i.e. $\dot{m}=3.25 \times 10^{-9} \mathrm{~m}^{3} / \mathrm{s}$ ) in a $105 \mathrm{ft}$ (i.e. $L=32.004 \mathrm{~m}$ ) long circular capillary with a diameter of 0.03 in (i.e. $D=7.62 \times 10^{-4} \mathrm{~m}$ ). In the 
1 beginning, the capillary is assumed filled with stationary oil without any initial asphaltene deposits

2 on its surface.

3 Properties and Sources/Sinks: In this example, the following values are used respectively for the oil

4 density, oil viscosity and deposit density: $\rho=820 \mathrm{~kg} / \mathrm{m}^{3}, \mu=3.95 \times 10^{-3} \mathrm{~kg} / \mathrm{m} \cdot \mathrm{s}$, and $\rho_{\text {dep }}=1200$

$5 \mathrm{~kg} / \mathrm{m}^{3}$. The temporally independent deposition rate is prescribed varying spatially as

$$
R_{\text {dep }}=A \frac{X}{L}+B
$$

where $A$ is a constant, $B$ is another constant and $L$ is the length of the capillary.

Exact Solutions: According to the prescribed deposition rate, the fluid volume fraction can be obtained analytically from

$$
\alpha(x, t)=\exp \left(-\frac{R_{d e p}}{\rho_{\text {dep }}} t\right)
$$

Besides, the crude oil volume fraction is defined as

$$
\alpha(x, t)=\frac{A_{i}}{A_{c}}=\frac{r_{i}^{2}}{R^{2}}
$$

where $R$ is the radius of the capillary. From eq 38, the deposition thickness $\delta$ is derived as

$$
\delta=R-r_{i}=R(1-\sqrt{\alpha})
$$

Therefore, the exact solution for the velocity is acquired as

$$
u(x, t)=\frac{u_{i n}}{\alpha(x, t)}+\left(\frac{1}{\rho}-\frac{1}{\rho_{d e p}}\right)\left(1+\frac{R_{d e p}}{\rho_{d e p}} t\right) \frac{\rho_{d e p}^{2}}{t^{2}} \frac{L}{A}
$$

The detailed derivation of eqs 37 and 40 is given in APPENDIX B.

Discussions of Results: For this demonstration, we set $A=0.2 \mathrm{~kg} / \mathrm{m}^{3}, B=0.02 \mathrm{~kg} / \mathrm{m}^{3}$ respectively. Figure 8 shows the deposition thickness and velocity plotted at every hour interval over a six hours period. The exact solutions are calculated using eqs 37 and 40 . The time step is set to $3.6 \mathrm{~s}$. The spatial domain is discretized into 10 and 100 CVs. As seen in Figure 8, the numerical solutions using coarse (10 CVs) and fine (100 CVs) spatial grids reproduced the exact solutions very well over the six hours. Figure 9 shows the effects of time steps. The spatial domain is discretized into 100 CVs. The results for time steps of $360 \mathrm{~s}$ and $36 \mathrm{~s}$ are shown. Note that from Figure 8, a time step of $3.6 s$ reproduced the exact solutions. It can be seen that a time step of $36 s$ also reproduces the exact solutions. With a time step of $360 \mathrm{~s}$, the deposition thickness is predicted quite well, especially with the scales in Figure 9. However, the errors in the velocity predictions are clearly observed. These errors accumulate and become significant as time passes. Although errors in the deposition thickness are small, the flow area covered by these errors is reasonably large leading to the large errors in the velocities. 
Remarks: This problem shows that the numerical procedure can predict the crude oil volume fraction, the deposition thickness and the fluid velocity when the deposition rate is known. For the problem tested, selection of time step sizes is more crucial than the spatial discretization. With grid (both spatial and temporal) refinements, the exact solutions are reproduced accurately. As this is a one-dimensional procedure, the solutions converge rapidly. This example shows the capability of the proposed procedure to model a very large aspect ratio $(L / D=42,000)$ capillary.

\section{Problem 4}

Problem Description: In this example, we use, as much as possible, the experimental parameters from the experiment of Wang et al. (2004). The oil containing $2.42 \%$ by weight of the dissolved asphaltene is mixed with precipitant $\left(\mathrm{C}_{15}\right)$ in a volumetric ratio of $76: 24$. Then, this oil-precipitant mixture flows at a constant volumetric flowrate of $11.7 \mathrm{ml} / \mathrm{hr}$ (i.e. $\dot{\mathrm{m}}=3.25 \times 10^{-9} \mathrm{~m}^{3} / \mathrm{s}$ ) in a $105 \mathrm{ft}$ (i.e. $L=32.004 \mathrm{~m}$ ) long circular capillary with a diameter of 0.03 in (i.e. $D=7.62 \times 10^{-4} \mathrm{~m}$ ). The capillary is initially filled with stationary oil and free of asphaltene deposition. The experiment was run for 35.9 hours. A non-destructive technique is used to measure the amount of asphaltene deposits formed along the capillary. Figure 10 shows the schematic of the experimental setup.

Properties and Sources/Sinks: The following values are also used in this example: $\rho=820 \mathrm{~kg} / \mathrm{m}^{3}$ and $\mu=3.95 \times 10^{-3} \mathrm{~kg} / \mathrm{m} \cdot \mathrm{s}$. the oil and precipitant densities are $\rho_{\text {oil }}=850 \mathrm{~kg} / \mathrm{m}^{3}$ and $\rho_{\text {precipitant }}=740$ $\mathrm{kg} / \mathrm{m}^{3}$ respectively. The density of asphaltene deposit is taken as $\rho_{\text {dep }}=1200 \mathrm{~kg} / \mathrm{m}^{3}$ (Rogel and Carbognani, 2003). The concentration of the dissolved asphaltene at the inlet of the capillary is calculated as $C_{d i s, i n}=2.42 \% \times 820 \times(0.76 \times 850) /(0.76 \times 850+0.24 \times 740) \approx 15.56 \mathrm{~kg} / \mathrm{m}^{3}$. Besides, we also assume that no precipitation occurs before the oil-precipitant mixture is fed to the capillary, i.e. $C_{\text {pre }, \text { in }}=0 \mathrm{~kg} / \mathrm{m}^{3}$.

The precipitation rate is modelled by eq 41 which accounts for both of the precipitation and redissolution phenomena (Vargas et al., 2010).

$$
R_{p r e}= \begin{cases}k_{p r e}\left(C_{d i s}-C_{e q}\right) & C_{d i s} \geq C_{e q} \\ -k_{d i s} C_{p r e} & C_{d i s}<C_{e q}\end{cases}
$$

where $k_{\text {pre }}$ is the precipitation rate constant, $k_{\text {dis }}$ is the dissolution rate constant, and $C_{\text {eq }}$ is the equilibrium concentration above which the dissolved asphaltene will precipitate out of the oil. The aggregation and deposition rates are modelled by

$$
\begin{aligned}
& R_{\text {agg }}=k_{\text {agg }} C_{p r e} \\
& R_{\text {dep }}=k_{\text {dep }} C_{p r e}
\end{aligned}
$$

where $k_{\text {agg }}$ is the aggregation rate constant and $k_{d e p}$ is the deposition rate constant. These first order rates are used for demonstration purpose. Other rate expressions can be used without new numerical treatments.

Discussions of Results: In this example, the rate constants determined by Kurup et al. (2011) are used directly: $k_{\text {pre }}=1.45 \times 10^{-3} \mathrm{~s}^{-1}, k_{\text {agg }}=5.07 \times 10^{-3} \mathrm{~s}^{-1}$ and $k_{\text {dep }}=1.31 \times 10^{-2} \mathrm{~s}^{-1}$. Besides, considering the fixed-temperature operating condition and very small pressure variation ( $0 \sim 27$ psi, Figure10), the thermodynamic modelling part is not required in this case. Therefore, for simplicity, $C_{e q}=0$ is set. If $C_{e q} \neq 0$, according to eq 41 , the amount of the precipitated asphaltene is smaller leading to a 
thinner asphaltene deposit layer. As for $k_{d i s}$, due to the lack of information in existing literature, it is also set to zero. If $k_{\text {dis }} \neq 0$ is used instead, because of re-dissolution, less precipitated asphaltene is potentially formed. In this situation, the predicted deposit layer thickness should be even lower. Consequently, it should be stressed here that under these constraints $\left(C_{e q}=0\right.$ and $\left.k_{d i s}=0\right)$, the numerical procedure presented in this article will overpredict asphaltene deposition in the capillary.

Figure 11 shows the mesh independent solution plotted against axial length $x$ by employing 400 CVs with a time step of $18 \mathrm{~s}$. For comparison purpose, the results obtained with $800 \mathrm{CVs}$ and $\Delta t=9$ $s$ are also included. As depicted in Figure 11a, a much thicker deposit is formed near the inlet and it becomes thinner downstream. This complies with the fact that the concentration of the precipitated asphaltene is higher around the inlet, and decreases along the capillary as deposition takes place consuming the amount of the precipitated asphaltene. As a result, a gradual lower deposition potential is caused towards the outlet of the capillary. The deposit layer thickness at $t=35.9 \mathrm{~h}$ is compared with the experiment result of Kurup et al. (2011) in Figure 11b. The prediction of the proposed numerical procedure agrees qualitatively with the experimental measurements. As explained, by setting $C_{e q}=0$ and $k_{d i s}=0$, the amount of asphaltene deposits is overpredicted for $x<$ $17.2 \mathrm{~m}$. However, the measured deposit profile in the near-exit-region is not captured well. The prediction shows that almost minute asphaltene deposit is formed toward the outlet of the capillary owing to the exhaustion of the precipitated asphaltene in the oil-precipitant flow. While the experiment result indicates a relatively higher amount of deposits with an almost constant thickness in the vicinity of the exit. This discrepancy may arise from the use of the constant rate coefficients in simulation, which may be insufficient to describe the processes occurring in the entire capillary. In addition, the shear removal of upstream deposits and re-deposition downstream are also not considered.

Remarks: In this problem, it is clearly seen that the fluid volume fraction, velocity and pressure fields vary with the occurrence and evolution of the asphaltene deposit layer. The proposed numerical procedure is able to couple the flow fields with the asphaltene precipitation, aggregation and deposition processes. Besides, it is demonstrated that the procedure presented in this article is capable of predicting the overall trend of the deposit profile, in particular, the location and magnitude of the maximum deposit layer thickness. The quite good agreement with the capillary asphaltene deposition measurements indicates the potential of this procedure to be applied for more complicated cases with large $L / D$ ratios, e.g. oilfield asphaltene problems, if accurate precipitation, dissolution, aggregation and deposition rate constants and the relevant thermodynamic information (e.g. $C_{e q}$ over a range of $p$ and $T$ conditions) are provided.

\section{CONCLUSIONS}

A numerical procedure within a unidirectional, one-dimensional, one-way framework is proposed to model asphaltene deposition in wellbores with large length-to-diameter ratios. In this article, we focus our attention only on the CFD part of the asphaltene deposition model. As such, the precipitation rate, aggregation rate and deposition rate are assumed either known or modelled using simple relations. Nevertheless, the procedure presented in this article is general. In other words, it can be used with other forms of rate expressions without additional numerical treatments.

Upon verification of the procedure, a few cases with known solutions are first studied. Then, this procedure is employed to predict asphaltene deposition in a capillary tube of which experimental data is available. Due to the uncertainty of the constant rate constants and the unavailability of some 
1 thermodynamic parameters, a qualitative agreement is obtained in terms of the overall trend of the

2 deposit layer profile along the capillary tube.

3 From these cases investigated, the following conclusions are made:

4 (1) The proposed numerical procedure is capable of predicting the pressure drop of both laminar 5 and turbulent flows in flow passages with large aspect ratios.

6 (2) The proposed procedure is able to account for the variation in the flow cross-sectional area, an 7 important parameter which is coupled with the velocity field, pressure field, and the precipitation, 8 aggregation and deposition processes.

9 (3) In the numerical procedure presented in this article, selection of time step sizes is more critical 10 than the spatial discretization. Besides, due to the one-dimensional nature of this procedure, the 11 convergence of solutions can be achieved readily and rapidly.

12 (4) The good agreement between the simulation results and the capillary experimental 13 measurements demonstrates that the proposed procedure can be used as a promising prediction tool 14 for investigation of oilfield asphaltene deposition problems.

This work is supported by a research grant from Abu Dhabi National Oil Company (ADNOC) R\&D Oil-Sub Committee. 
Table 1: Governing equations and meanings of various terms.

\begin{tabular}{ccccc}
\hline Equation & $\phi$ & $\tilde{\rho}$ & $S_{\phi}$ & Equation \\
\hline Continuity & 1 & $\rho$ & $-R_{d e p}$ & $(1)$ \\
Momentum & $u$ & $\rho$ & $-\frac{d p}{d x}-F_{w}+S_{u}$ & $(2)$ \\
Dissolved Asphaltene & $C_{\text {dis }}$ & 1 & $-R_{p r e}+S_{d i s}$ & $(3)$ \\
Precipitated Asphaltene & $C_{p r e}$ & 1 & $R_{p r e}-R_{a g g}-R_{d e p}+S_{p r e}$ & $(4)$ \\
Energy & $T$ & $\rho c_{p}$ & $S_{T}$ & $(5)$ \\
\hline
\end{tabular}

3

Table 2: Laminar friction constant $C$ for ducts of various shapes (White, 2003).

\begin{tabular}{|c|c|c|c|c|c|c|}
\hline Circular & \multirow{2}{*}{\multicolumn{2}{|c|}{$\underset{b / a}{C}$}} & \multicolumn{2}{|c|}{ 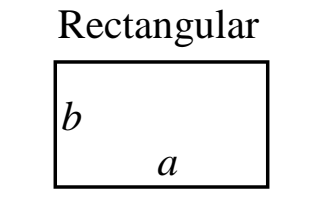 } & \multicolumn{2}{|c|}{$\underbrace{\text { Isosceles Triangle }}_{\uparrow 2 \theta}$} \\
\hline$C$ & & & $b / a$ & $C$ & $\theta$ (deg) & C \\
\hline 64.00 & $\begin{array}{c}0 \\
0.00001 \\
0.0001 \\
0.001 \\
0.01 \\
0.05 \\
0.1 \\
0.2 \\
0.4 \\
0.6 \\
0.8 \\
1\end{array}$ & $\begin{array}{l}64.00 \\
70.09 \\
71.78 \\
74.68 \\
80.11 \\
86.27 \\
89.37 \\
92.35 \\
94.71 \\
95.59 \\
95.92 \\
96.00 \\
\end{array}$ & $\begin{array}{c}0 \\
0.05 \\
0.10 \\
0.125 \\
0.167 \\
0.25 \\
0.4 \\
0.5 \\
0.75 \\
1\end{array}$ & $\begin{array}{l}96.00 \\
89.91 \\
84.68 \\
82.34 \\
78.81 \\
72.93 \\
65.47 \\
62.19 \\
57.89 \\
56.91\end{array}$ & $\begin{array}{c}0 \\
10 \\
20 \\
30 \\
40 \\
50 \\
60 \\
70 \\
80 \\
90\end{array}$ & $\begin{array}{l}48.00 \\
51.60 \\
52.90 \\
53.30 \\
52.90 \\
52.00 \\
51.10 \\
49.50 \\
48.30 \\
48.00\end{array}$ \\
\hline
\end{tabular}

Table 3: Re, $f_{D}$ and theoretical major pressure drop in the three pipe sections.

\begin{tabular}{cccc}
\hline $\begin{array}{c}\text { Section } \\
\text { No. }\end{array}$ & $\operatorname{Re}$ & $f_{D}$ & $\begin{array}{c}(\Delta p)_{\text {major }} \\
(\mathrm{Pa})\end{array}$ \\
\hline 1 & 830.38 & 0.07707 & 632.00 \\
2 & 1660.76 & 0.03854 & 5056.00 \\
3 & 2372.51 & 0.04683 & 7311.67 \\
\hline
\end{tabular}


Figure 1: Ingredients of an asphaltene deposition model.

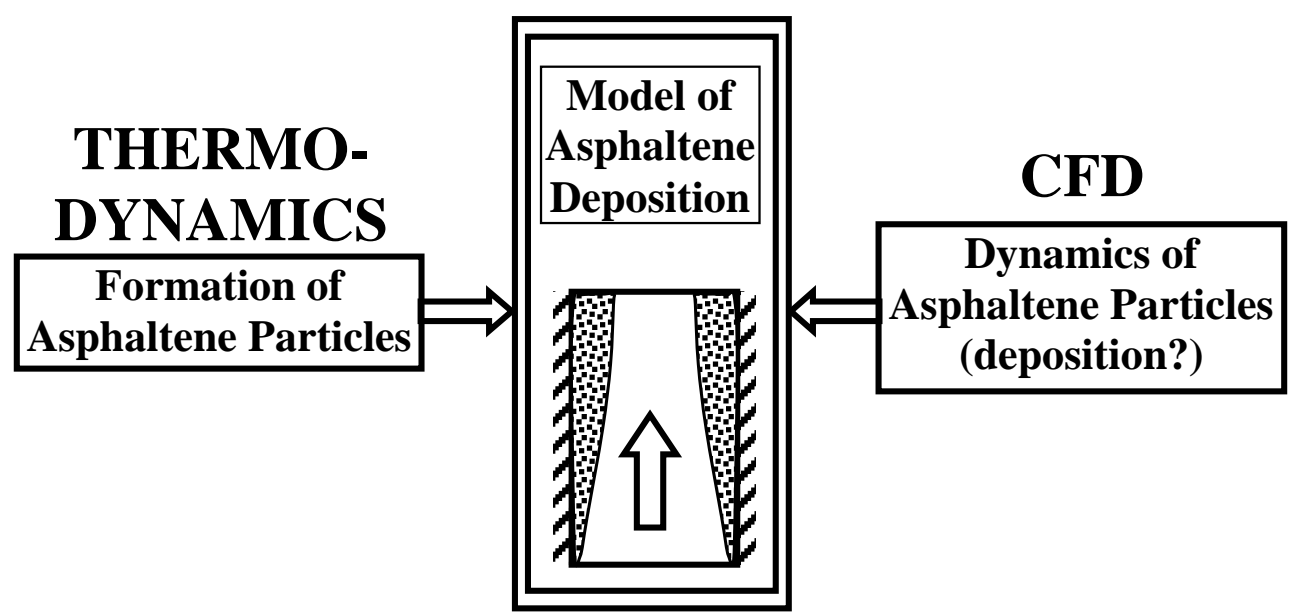

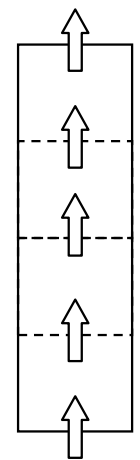

(a)

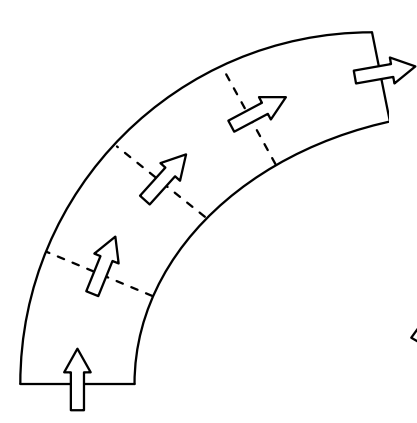

(b)

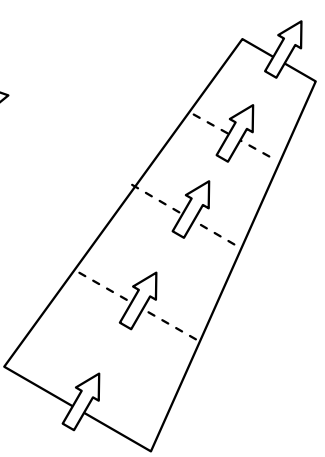

(c)

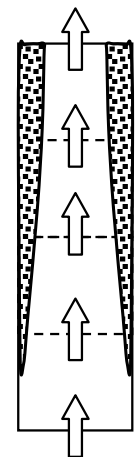

(d)

Figure 2: Unidirectional, one-dimensional, one-way flows.

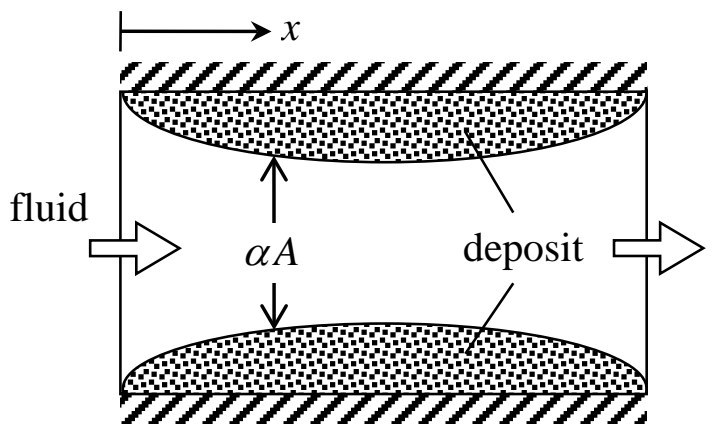

(a)

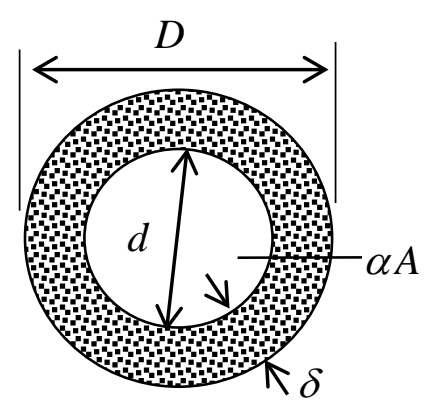

(b)
8 


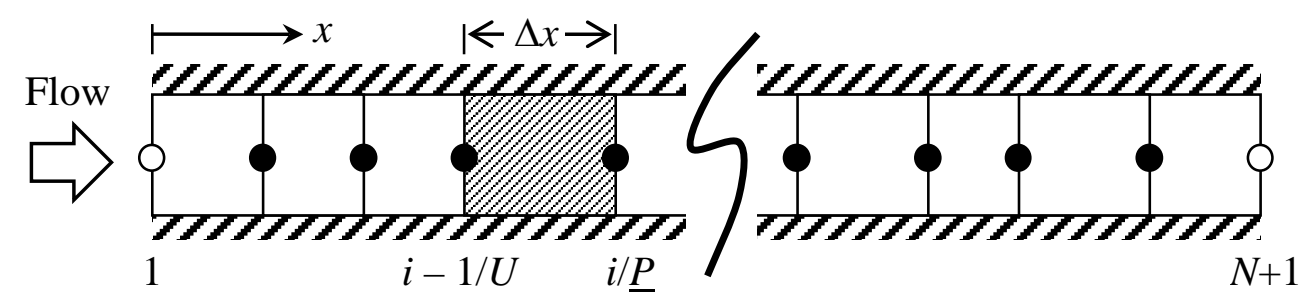

Figure 4: Schematic of the discretized computational domain.

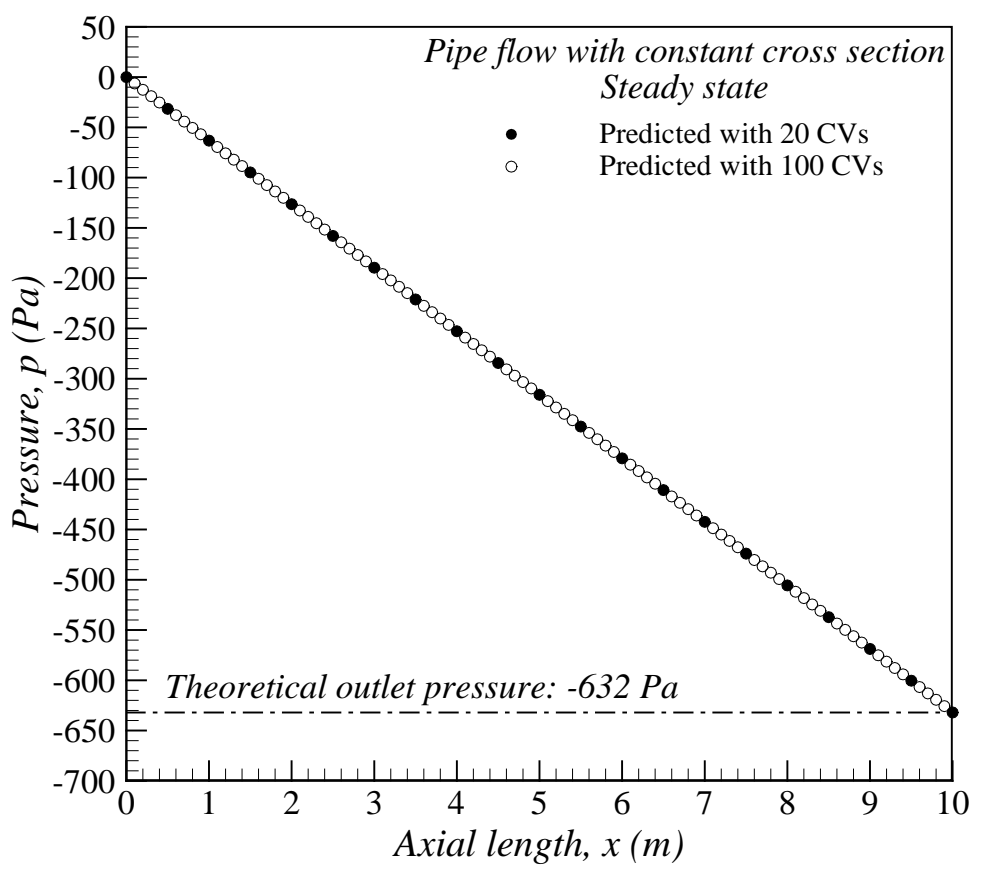

Figure 5: Pressure drop prediction for pipe flow of constant cross section.

6

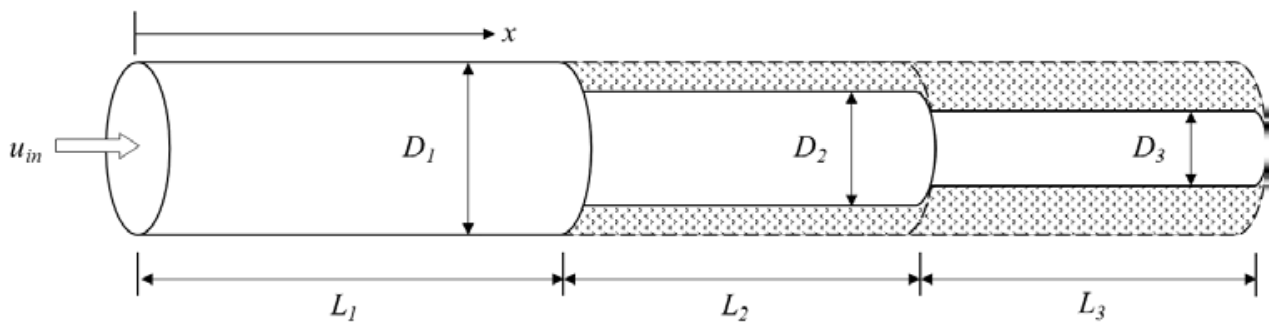

Figure 6: Schematic of the pipe flow with variable cross section. 


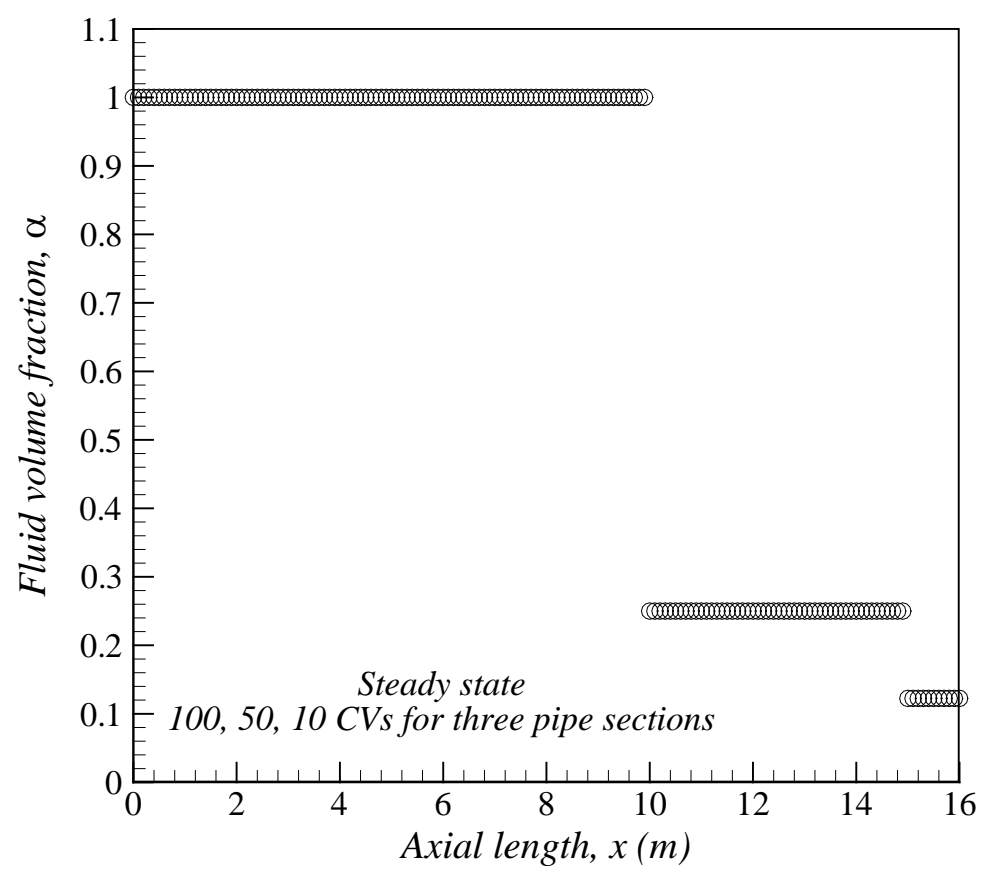

(a) fluid volume fraction

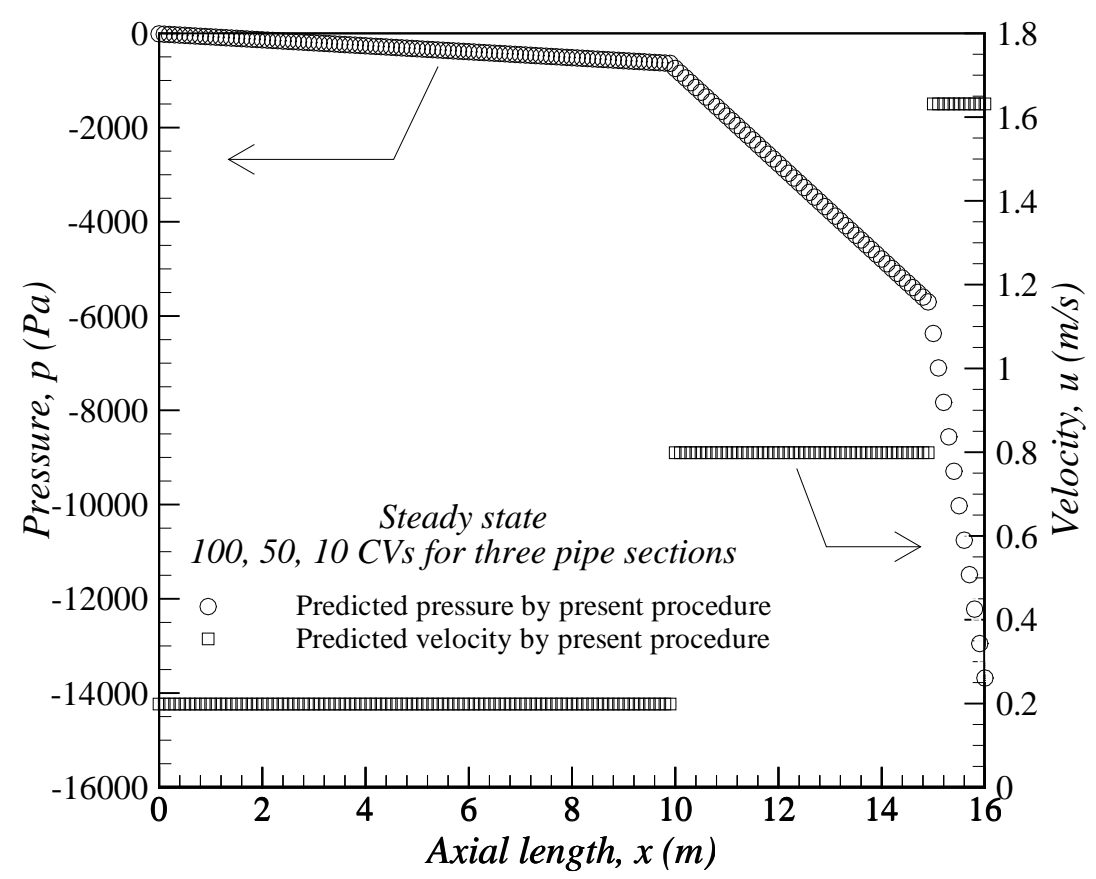

(b) pressure and velocity

$5 \quad$ Figure 7: Fluid volume fraction, pressure and velocity for pipe flow of variable cross section. 


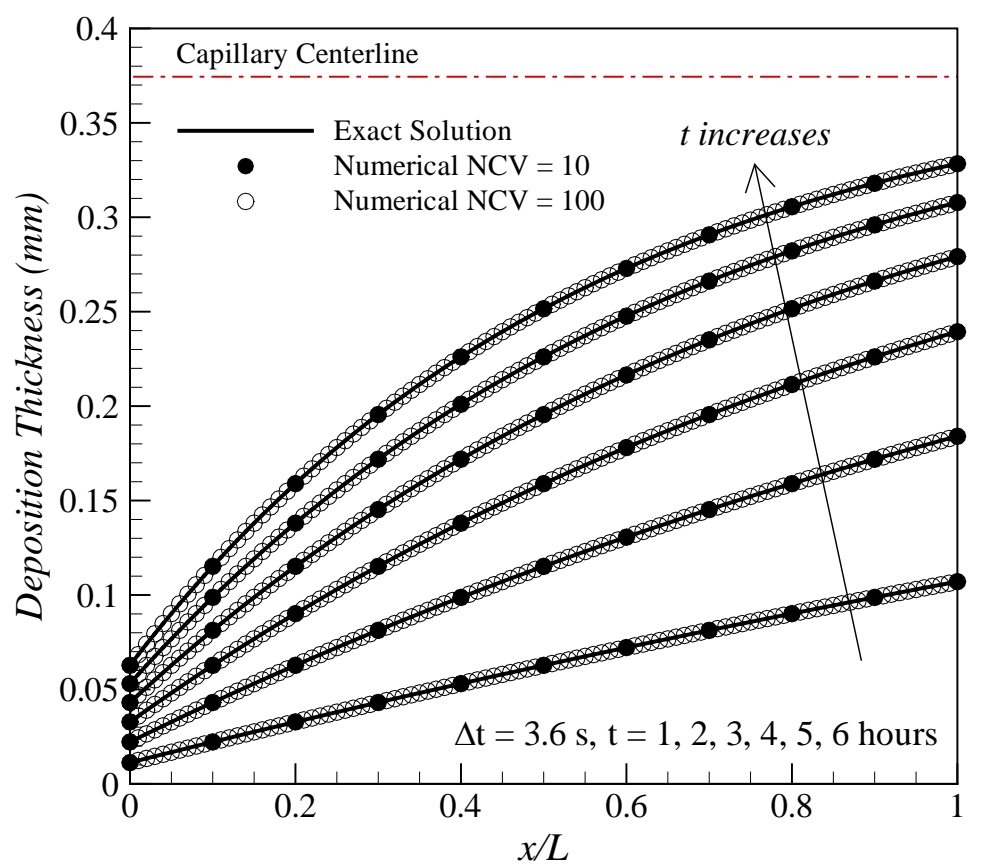

(a) deposition thickness

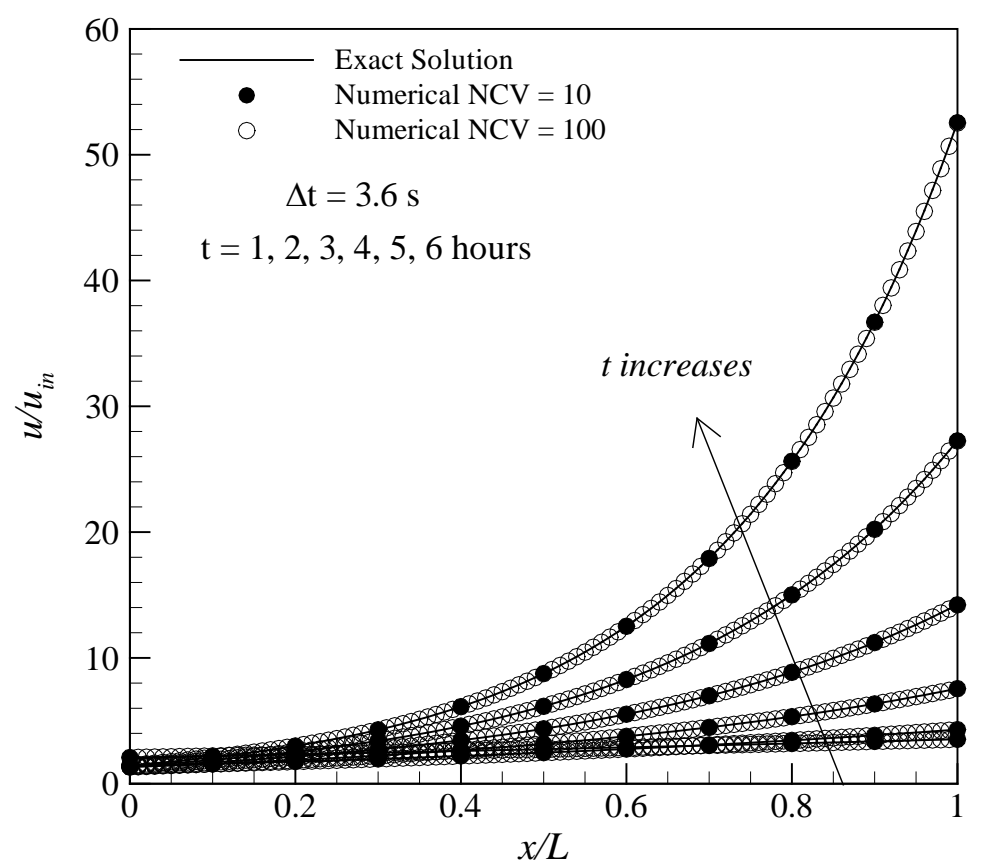

(b) relative velocity

Figure 8: Effects of number of control volumes with time step of $3.6 \mathrm{~s}$. 


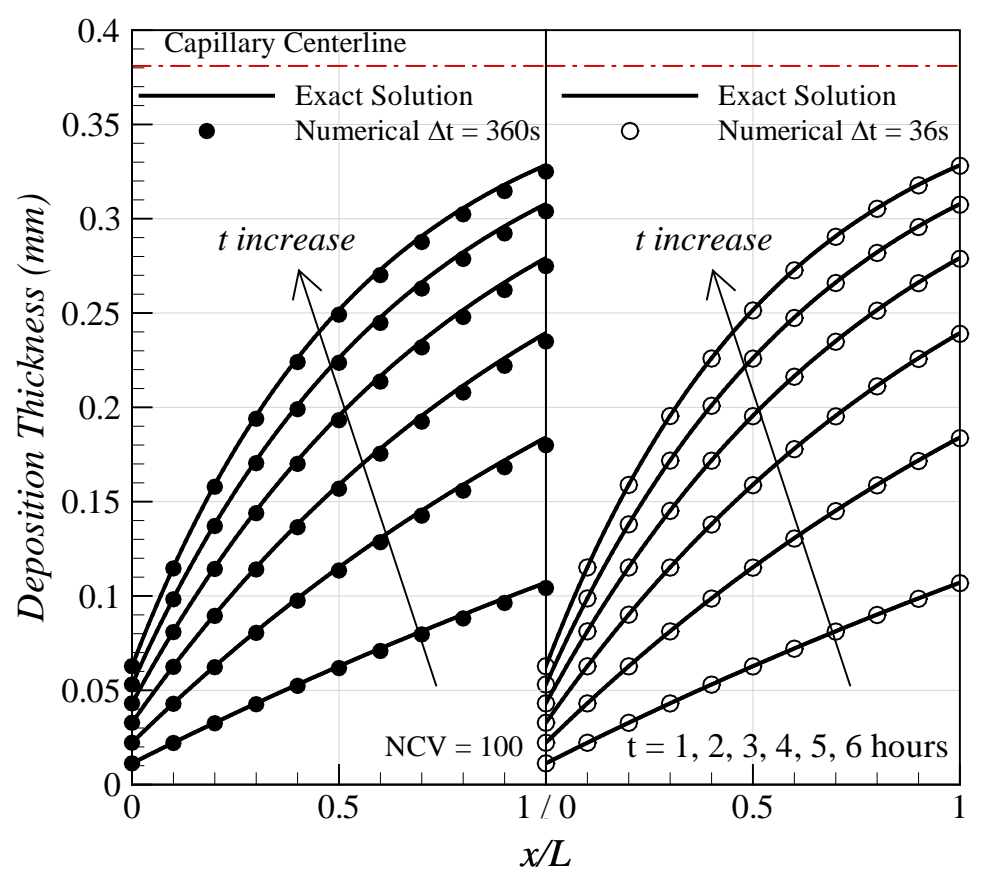

(a) deposition thickness

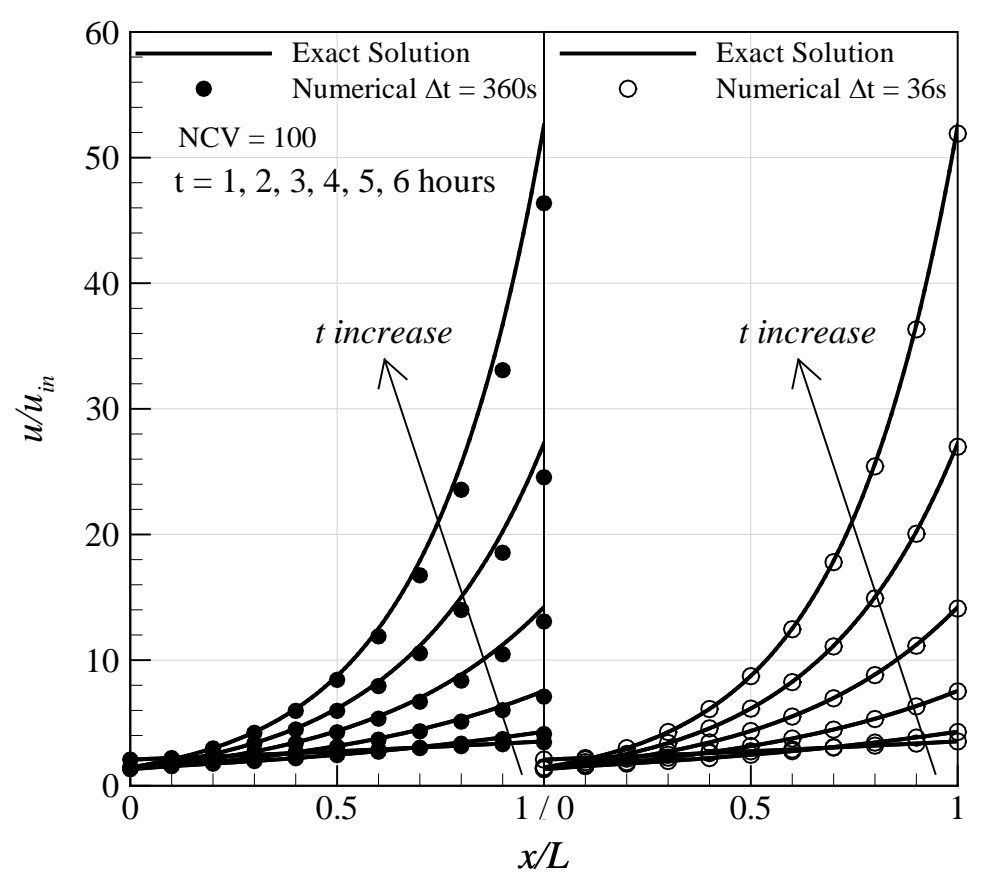

(b) relative velocity

Figure 9: Effects of time step with 100 spatial control volumes. 


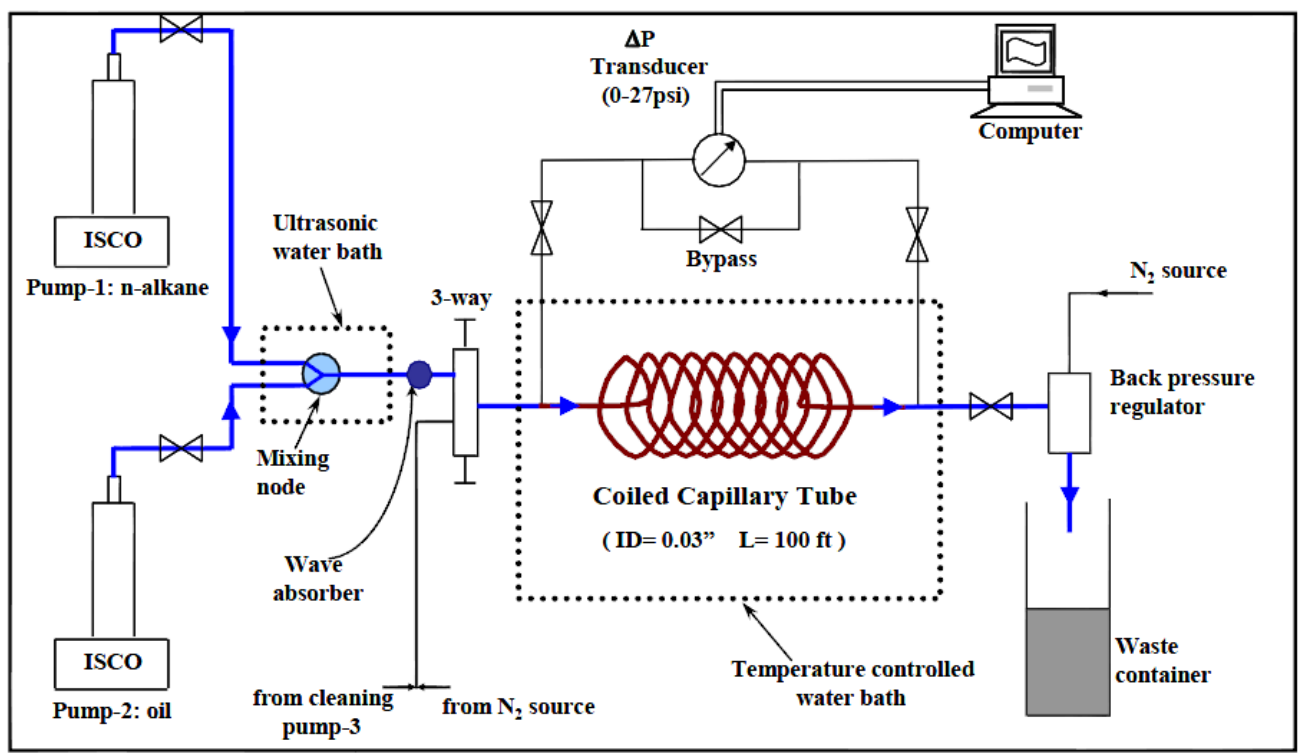

Figure 10: Schematic of the capillary deposition experimental setup (Kurup et al., 2011). 


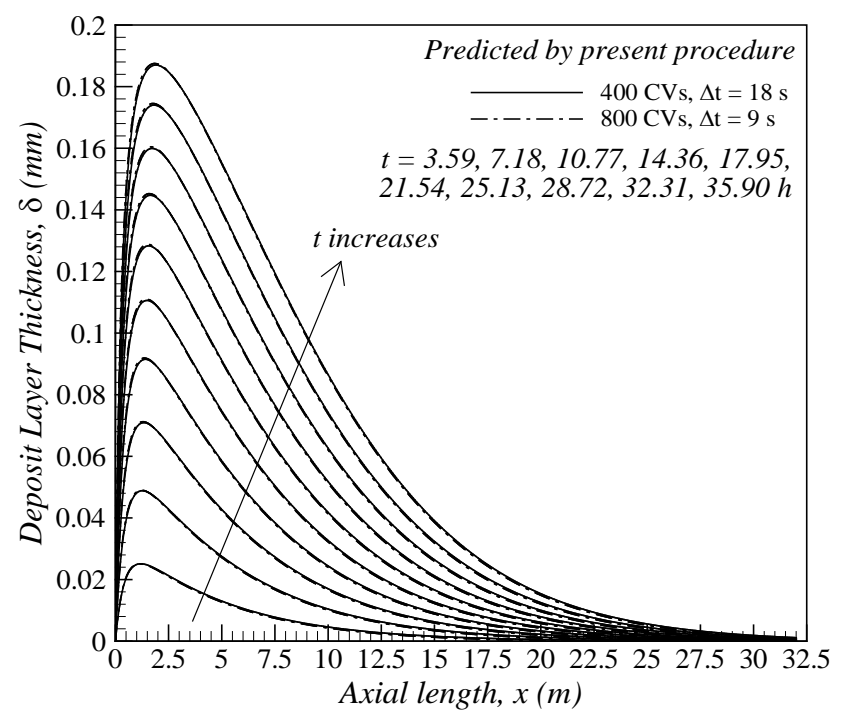

(a) deposit layer thickness

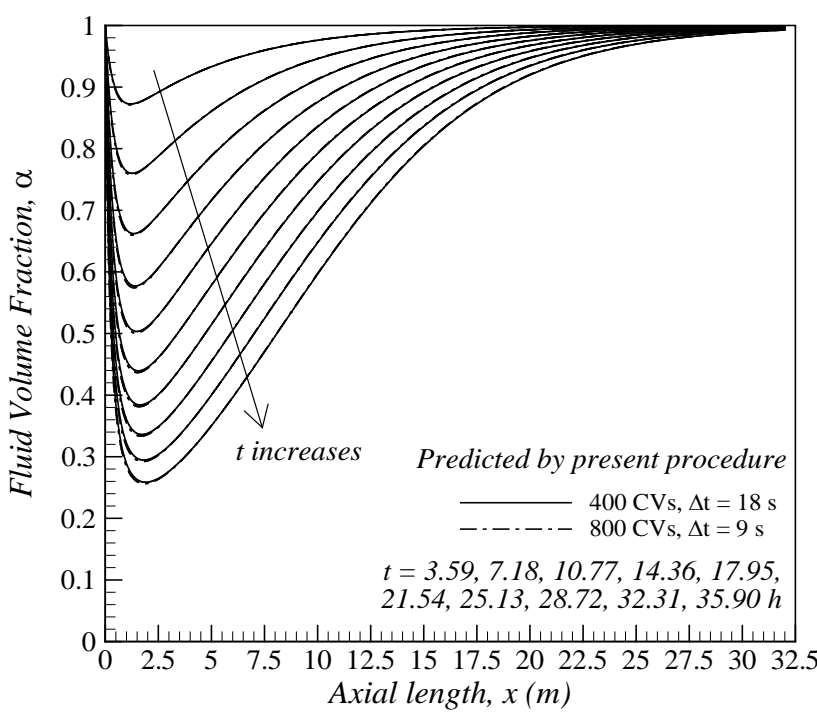

4

(c) fluid volume fraction

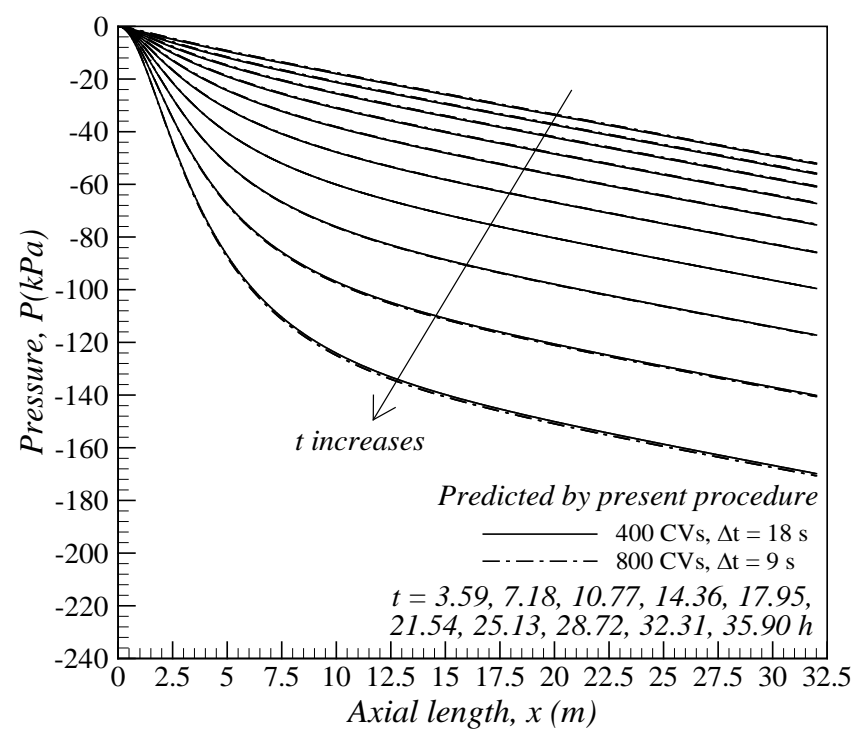

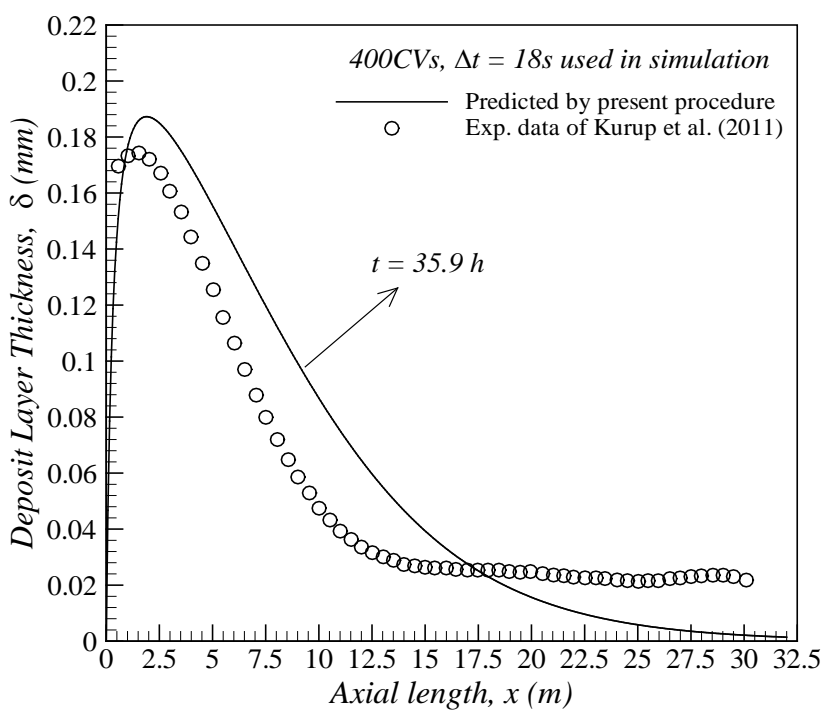

(b) comparison with experiment data

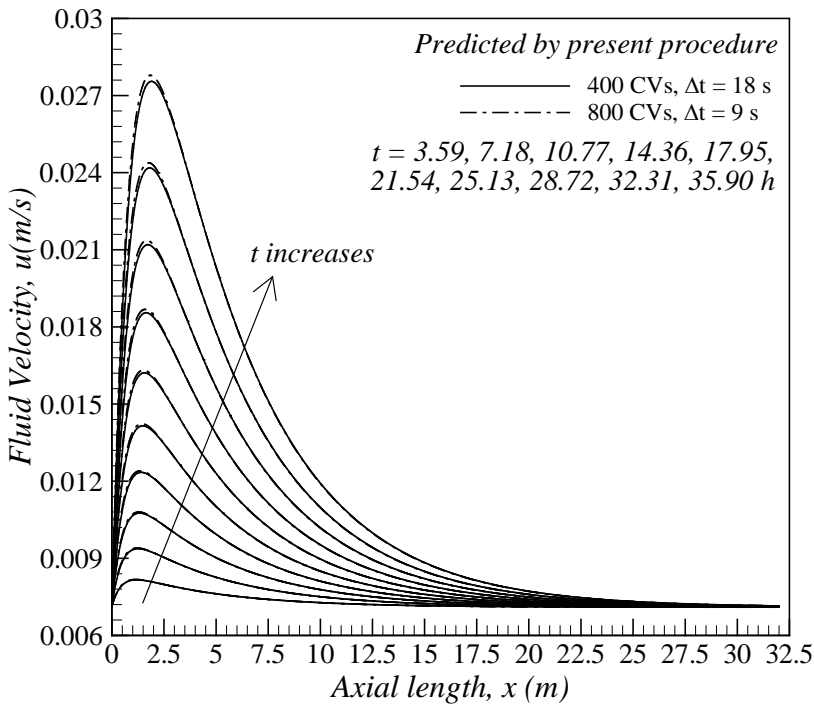

(d) fluid velocity

(e) pressure

Figure 11: Simulation results of the capillary asphaltene deposition experiment 


\section{A. Derivation of the continuity equation}

or

$$
\frac{\partial}{\partial t}(\rho \alpha)+\frac{\partial}{\partial x}(\alpha \rho u)=-\alpha R_{d e p}
$$

For an elemental control volume (CV) shown in Figure A1, the conservation of mass can be expressed mathematically as

$$
\frac{\partial}{\partial t} \int_{C V} \rho d V_{f}+\oint_{C S} \rho \vec{u} \cdot d \vec{S}_{f}=-\int_{C V} R_{d e p} d V_{f}
$$

where $\rho$ and $\vec{u}$ are respectively the fluid density and the fluid velocity vector, and $R_{d e p}$ is the volumetric rate of deposition. The subscript ' $f$ ' stands for the property associated with the flow passage. In this expression, the first term on the left-hand-side represents the rate of change of mass within the CV. The second term on the left-hand-side accounts for the net convective transport of mass out of the CV via the control surface (CS). The term on the right-hand-side is a mass sink to account for the particles consumed from the flowing fluid during the deposition process. It should be stressed here that, due to the existence of a deposit layer, the volume and surface integrations are performed only with respect to the flow passage rather than the entire channel. The transient quasione-dimensional form of eq A1 is sought here. By defining $\alpha$ (the crude oil volume fraction) as the ratio of the volume of flow passage to the total volume of a $\mathrm{CV}$, i.e. $\alpha=V_{f} / V_{C V}$, for a small $d x$, we have $d V_{f}=\alpha d V_{C V}=\alpha A_{c} d x$ and therefore eq A1 can be approximately evaluated as

$$
\begin{gathered}
\frac{\partial}{\partial t}\left(\rho \alpha A_{c} d x\right)+\left(\rho u \alpha A_{c}\right)_{x+\Delta x}-\left(\rho u \alpha A_{c}\right)_{x}=-R_{d e p} \alpha A_{c} d x \\
\frac{\partial}{\partial t}\left(\rho \alpha A_{c} d x\right)+\frac{\partial}{\partial x}\left(\rho u \alpha A_{c}\right) d x=-R_{d e p} \alpha A_{c} d x
\end{gathered}
$$

\section{B. Derivation of the exact solution of $\alpha$ and $u$ under the given $R_{d e p}$}

Since the asphaltene deposition rate is prescribed as 


$$
R_{\text {dep }}=A \frac{X}{L}+B
$$

1 the change in the mass of deposit over a time step $d t$ is

$$
\frac{d M_{d e p}}{d t}=\int_{x_{i-1}}^{x_{i}} \alpha R_{d e p} A_{c} d x=\int_{x_{i-1}}^{x_{i}} \alpha\left(A \frac{x}{L}+B\right) A_{c} d x
$$

2 Assume that $a$ is constant over a control volume eq B2 becomes

$$
\frac{d\left\lfloor\rho_{\text {dep }}(1-\alpha) A_{c} \Delta x\right\rfloor}{d t}=\alpha A_{c} \int_{x_{i-1}}^{x_{i}}\left(A \frac{x}{L}+B\right) d x
$$

3 or

$$
-\rho_{\text {dep }} A_{c} \Delta x \frac{d \alpha}{d t}=\alpha A_{c} \Delta x\left(A \frac{X_{i-1}+x_{i}}{2 L}+B\right)
$$

$4 \quad$ Rearranging eq B4 and integrating with the proper limits gives

$$
\int_{1}^{\alpha} \frac{d \alpha}{\alpha}=\int_{0}^{t} \frac{-1}{\rho_{d e p}}\left(A \frac{x_{i-1}+x_{i}}{2 L}+B\right) d t
$$

5 or

$$
\ln \alpha=\frac{-1}{\rho_{\text {dep }}}\left(A \frac{X_{i-1}+X_{i}}{2 L}+B\right) t
$$

$6 \quad$ Eq B6 can be written as

$$
\alpha=\exp \left(\frac{-1}{\rho_{\text {dep }}}\left[\frac{A}{L} x+B-\frac{A \Delta x}{2 L}\right] t\right)
$$

$7 \quad$ Provided $\Delta x$ is sufficiently small, eq B7 simplifies as

$$
\alpha=\exp \left(\frac{-1}{\rho_{\text {dep }}}\left[\frac{A}{L} x+B\right] t\right)=\exp \left(\frac{-R_{d e p}}{\rho_{\text {dep }}} t\right)
$$

8 According to eq B8,

$$
\frac{\partial \alpha}{\partial t}=-\frac{\alpha R_{d e p}}{\rho_{d e p}}
$$

9 The continuity equation is

$$
\frac{\partial}{\partial t}(\alpha \rho)+\frac{\partial}{\partial x}(\alpha \rho u)=-\alpha R_{d e p}
$$


1 Under the constant fluid density assumption,

$$
\rho \frac{\partial \alpha}{\partial t}+\rho \frac{\partial}{\partial x}(\alpha u)=-\alpha R_{d e p}
$$

2 Subsisting eq B9 into eq B11 and rearranging it gives

$$
\frac{\partial}{\partial x}(\alpha u)=\alpha R_{d e p}\left(\frac{1}{\rho_{\text {dep }}}-\frac{1}{\rho}\right)
$$

3 or

$$
d(\alpha u)=\alpha R_{d e p}\left(\frac{1}{\rho_{\text {dep }}}-\frac{1}{\rho}\right) d x
$$

4 Integrate both sides with proper limits,

$$
\int_{u_{i n}}^{\alpha u} d(\alpha u)=\int_{0}^{x} \alpha R_{d e p}\left(\frac{1}{\rho_{d e p}}-\frac{1}{\rho}\right) d x=\left(\frac{1}{\rho_{d e p}}-\frac{1}{\rho}\right) \int_{0}^{x} R_{d e p} \alpha d x
$$

5 From eq B8,

$$
\frac{\partial \alpha}{\partial x}=\alpha\left(-\frac{A t}{L \rho_{\text {dep }}}\right)
$$

6 Therefore,

$$
\alpha d x=\left(-\frac{L \rho_{\text {dep }}}{A t}\right) d \alpha
$$

$7 \quad$ Substituting eq B16 into eq B14 yields

$$
\begin{gathered}
\alpha u-u_{\text {in }}=\left(\frac{1}{\rho_{\text {dep }}}-\frac{1}{\rho}\right)\left(-\frac{L \rho_{\text {dep }}}{A t}\right) \int_{1}^{\alpha} R_{d e p} d \alpha \\
\alpha u-u_{\text {in }}=\left(\frac{1}{\rho_{\text {dep }}}-\frac{1}{\rho}\right)\left(-\frac{L \rho_{\text {dep }}}{A t}\right)\left[R_{d e p} \alpha-\int_{B}^{\frac{A x}{L}+B} \alpha d\left(R_{d e p}\right)\right] \\
\alpha u-u_{\text {in }}=\left(\frac{1}{\rho_{\text {dep }}}-\frac{1}{\rho}\right)\left(-\frac{L \rho_{\text {dep }}}{A t}\right)\left[R_{d e p} \alpha-\frac{A}{L} \int_{0}^{x} \alpha d x\right] \\
\alpha u-u_{i n}=\left(\frac{1}{\rho_{\text {dep }}}-\frac{1}{\rho}\right)\left(-\frac{L \rho_{d e p}}{A t}\right)\left[R_{d e p} \alpha-\frac{A}{L}\left(-\frac{L \rho_{d e p}}{A t}\right) \alpha\right]
\end{gathered}
$$




$$
\alpha u-u_{i n}=\alpha\left(\frac{1}{\rho}-\frac{1}{\rho_{d e p}}\right)\left(\frac{L \rho_{d e p}^{2}}{A t^{2}}\right)\left(1+\frac{R_{d e p}}{\rho_{d e p}} t\right)
$$

1 Finally, the exact solution of $u$ is obtained as

$$
u=\frac{u_{i n}}{\alpha}+\left(\frac{1}{\rho}-\frac{1}{\rho_{d e p}}\right)\left(1+\frac{R_{d e p}}{\rho_{d e p}} t\right) \frac{\rho_{d e p}^{2}}{t^{2}} \frac{L}{A}
$$

2

3 
Kor, P. and Kharrat, R., 2016. Prediction of the Asphaltene Deposition Profile along a Wellbore During Natural Production from a Reservoir. Energy Sources, Part A: Recovery, Utilization, and Environmental Effects, 38(19): 2837-2844.

Kurup, A.S. et al., 2011. Development and Application of an Asphaltene Deposition Tool (ADEPT) for Well Bores. Energy \& Fuels, 25(10): 4506-4516.

Kurup, A.S. et al., 2012. Revisiting Asphaltene Deposition Tool (ADEPT): Field Application. Energy \& Fuels, 26(9): 5702-5710.

Mansoori, G.A., 2010. Remediation of Asphaltene and Other Heavy Organic Deposits in Oil Wells and in Pipelines. SOCAR Proceedings, 2010(4): 12-23.

Mukherjee, H. and Brill, J., 1985. Pressure Drop Correlations for Inclined Two-Phase Flow. Journal of Energy Resources Technology, 107(4): 549-554.

Nghiem, L., Hassam, M., Nutakki, R. and George, A., 1993. Efficient Modelling of Asphaltene Precipitation, 68th SPE Annual Technical Conference and Exhibition. Society of Petroleum Engineers, Houston, Texas, US.

Patankar, S., 1980. Numerical Heat Transfer and Fluid Flow. CRC press.

Ramey Jr, H., 1962. Wellbore Heat Transmission. Journal of Petroleum Technology, 14(04): 427435.

Ramirez-Jaramillo, E., Lira-Galeana, C. and Manero, O., 2006. Modeling Asphaltene Deposition in Production Pipelines. Energy \& Fuels, 20(3): 1184-1196.

Rogel, E. and Carbognani, L., 2003. Density Estimation of Asphaltenes using Molecular Dynamics Simulations. Energy \& Fuels, 17(2): 378-386.

Soulgani, B.S., Rashtchian, D., Tohidi, B. and Jamialahmadi, M., 2009. Integrated Modelling Methods for Asphaltene Deposition in Wellstring. Journal of the Japan Petroleum Institute, 52(6): 322-331322.

Vargas, F.M., Creek, J.L. and Chapman, W.G., 2010. On the Development of an Asphaltene Deposition Simulator. Energy \& Fuels, 24(4): 2294-2299.

Wang, J., Buckley, J.S. and Creek, J.L., 2004. Asphaltene Deposition on Metallic Surfaces. Journal of Dispersion Science and Technology, 25(3): 287-298.

White, F.M., 2003. Fluid Mechanics. 5th. Boston: McGraw-Hill Book Company. 